\title{
Nuclear Fission Induced by Pi Mesons
}

\author{
R. J. Peterson* \\ Department of Physics, UCB 390, University of Colorado, Boulder CO 80309, USA
}

\author{
Received: April 21, 2010; In Final Form: June 14, 2010
}

\begin{abstract}
Pi mesons are an important part of the interactions among strongly-interacting particles, and their reactions with complex nuclei involve reactions with a wide range of interactions and time scales, with the slowest being the familiar fission process. Decades of effort have produced a wide range of fission data with stopped and energetic charged beams, both positive and negative. These results are the result of many uncoordinated studies, but in total can give a very good view of pion-induced fission. This review will compare and combine the measurements, with comparisons to a range of theoretical expectations. It is found that the nature of fission induced by pi mesons is not significantly different from fission induced by other energetic particles, in spite of the special features of the mesonic beam. This specific arena of nuclear science may now be considered complete.
\end{abstract}

\section{Introduction}

A range of observables for fission events resulting from irradiation of complex nuclei with beams of charged pi $(\pi)$ mesons (pions) has been obtained over the last half century, including a campaign of systematic studies using the intense beams from 'meson factories' and an efficient detection technique. This effort is now complete. The data arise from a variety of techniques and experimental groups, each with specific features. This review will bring together these data, with comparisons to seek consistency among the data, connections to the special field of pion-nucleus reactions, and comparisons to fission induced by photons and antiprotons.

1.1. Definitions of fission. The collision of an energetic beam particle with a complex nucleus may lead to heavy fragments by several processes. Spallation, with several lighter fragments, may produce a single heavy residue, or an energetic multifragmention reaction may shatter the target nucleus into several massive fragments. Both of these processes need to be distinguished from true fission, in which two (rarely three) fragments of not-too-different mass are formed from the same scission, with a Coulomb repulsion as the main source of their kinetic energy. ${ }^{1}$ Some of the experimental methods sense only one of a presumed pair of fragments. Since methods have different sensitivities to fragment properties, intercomparisons of results must be done with care. In this work 'fission' is defined to be the detection of one or two fragments, each with near half the target mass, and with energies as appropriate to the Coulomb repulsion of fission. Representative data demonstrating this selection process will be found in Section 3.

1.2. Pion-nucleus reactions. Pi mesons are fields, and may be absorbed into complex nuclei, making available their kinetic plus rest mass $(140 \mathrm{MeV})$ energies and their charge (plus or minus for beams), with little angular momentum due to the low beam mass. Pions must be absorbed onto two or more nucleons in their initial interaction (in order to conserve both energy and momentum), and these absorption cross sections can be a large fraction of the total reaction cross sections on heavy nuclei. ${ }^{2}$ Stopped $\pi$ may also be captured into a heavy nucleus from atomic orbits, with only the pion rest mass as the energy available for reactions leading to fission. A very complete comparison of theory and data (not including fission) for

*Corresponding author. E-mail: Jerry.Peterson@ Colorado.EDU nuclear reactions following the capture of negative pions $\left(\pi^{-}\right)$is found in Reference 3.

Since energetic pions interact with free nucleons by a series of important resonances, the energy dependence of the total reaction cross section $\left(\sigma_{\mathrm{R}}\right)$, of which absorption and fission will be a part, is a starting point for this review. Figure 1 shows reaction cross section data for pion beams of both signs on lead or bismuth up to kinetic energies of $2500 \mathrm{MeV}$. For comparison, the free negative pion-nucleon total cross sections, summed for the nucleons in lead, are also shown to exhibit the resonances. ${ }^{10}$ These structures are severely damped and quenched within a complex nucleus, as discussed in the review of Reference 2. Examples of computed reaction cross sections are shown, using the Distorted Wave Impulse Approximation (DWIA) $^{8}$ for both signs up to $300 \mathrm{MeV}$, showing a strong Coulomb effect, and an eikonal optical model at higher energies. ${ }^{9}$ These computed reaction cross sections $\sigma_{R}$ will form the denominators of fission probabilities in Section 7 below.

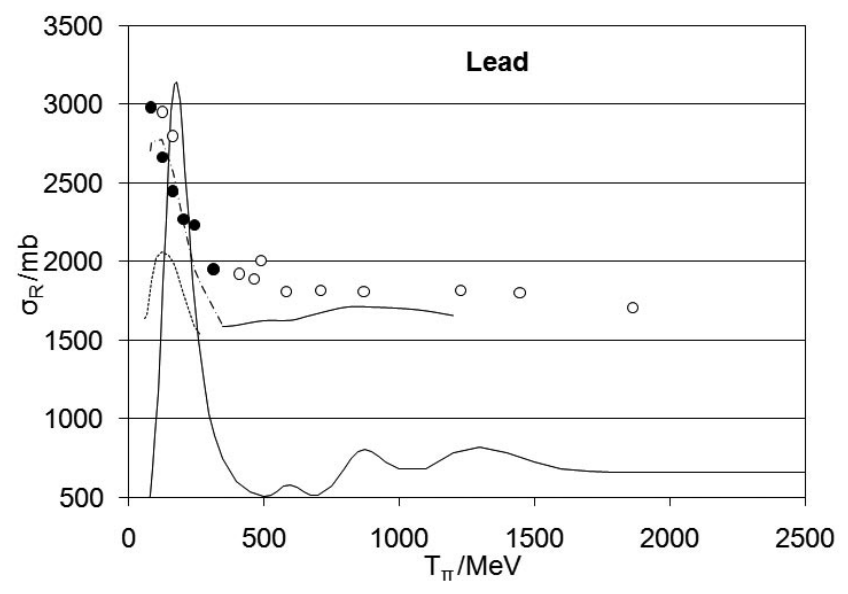

Figure 1. Pion-lead reaction cross sections $\sigma_{R}$ are shown, with data as collected in References 4-7. Open points are for $\pi^{-}$, solid for $\pi^{+}$. The DWIA calculations for $\pi^{+}$are dashed, with dot-dash for $\pi^{-8}$ An eikonal distorted wave model at higher energies for $\pi^{-}$gives the upper solid curve. ${ }^{9}$ The resonant structure for pions is shown by the negative pion-nucleon total cross sections ${ }^{10}$ summed for lead and divided by ten, as the more structured curve; these structures are smoothed out and damped in reactions with a heavy nucleus. These reaction cross sections form the denominator for fission probability calculations. For comparison, a black disk of lead with radius $1.3 A^{1 / 3} \mathrm{fm}$ has a cross section of $1864 \mathrm{mb}$. 
The measured beam energy dependence of absorption cross sections $\left(\sigma_{\mathrm{abs}}\right)$ of pions on bismuth, as a fraction of the total reaction cross section $\left(\sigma_{\mathrm{R}}\right)$, is shown in Figure 2, rising to $84 \%$ at $315 \mathrm{MeV}$. At the resonant energy of $165 \mathrm{MeV}$, Figure 3 shows the $\pi^{+}$absorption cross sections as a fraction of the reaction cross section as a function of target mass. The rise for heavier nuclei is evident. Both figures use the smooth fits to data shown in Reference 2. Evidence will be shown below that such absorption does indeed lead to fission. At low pion energies on gold, $\pi^{-}$absorption cross sections are twice those for $\pi^{+}$, but these yields are nearly equal by $250 \mathrm{MeV}{ }^{2}$ This is a Coulomb effect, with negative pions drawn into the delta resonance. By a beam energy of $500 \mathrm{MeV}$, pion absorption on a heavy nucleus with the emission of at least three protons is the dominant process. ${ }^{11}$

A wide range of de-excitations will follow the initial collision and likely absorption. Indicators of highly energetic processes have been presented by Nakai et al., in a measurement of the multiplicities of protons arising from 2,3 , and $4 \mathrm{GeV}$ pions stopping within the target nucleus. ${ }^{12,13}$ At least $30 \%$ of these reactions form a highly energetic moving source of the observed protons. In the work of Reference $14,5-15 \mathrm{GeV}$ beams of $\pi^{-}$and protons on gold found the distributions of charged particles and intermediate mass fragments to be insensitive to the beam species or momentum, indicating that the excitation energy to the target nucleus saturates. At $\Delta$ resonant energies near $150 \mathrm{MeV}$, McKeown et al. ${ }^{15}$ found that about 5.5 nucleons are involved in pion absorption on tantalum. Since the $\Delta$ formed by a pion-nucleon interaction interacts with further nucleons, a counting system leads to the expectation of four energetic nucleons with prompt connections to the pion. ${ }^{16}$ Golubeva et al. considered a moving source model, in which a $\pi^{+}$incident upon copper forms an excited region in the target nucleus. ${ }^{17}$ The number of involved nucleons rises from four at low energies to seventeen for pions of several GeV. All of these results indicate that an initial state of high excitation shared among a number of nucleons can be formed from an energetic pion beam incident upon a heavy nucleus.

The slowest and last nuclear de-excitation will be fission. Might some of the initial high nuclear excitation be noted in cross sections for fission induced by pi meson beams? Although this will be a question examined in detail below, the nonobservation of the inverse reaction, pion production during spontaneous fission, ${ }^{18}$ suggests that fission from an extreme state with the pion mass directly coupled to fission is not to be expected.

Since a direct path from pion beams and an initial very high excitation to fission does not exist, fission events will follow a

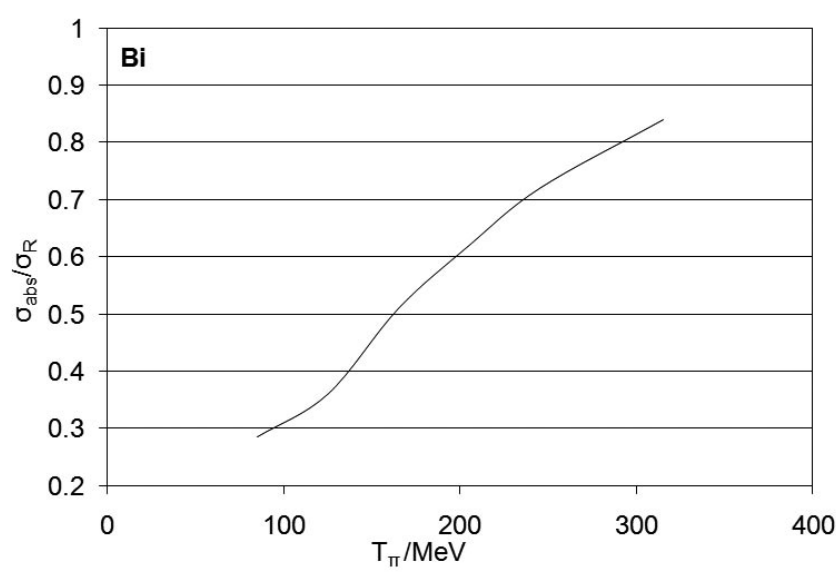

Figure 2. Measured pion-bismuth absorption and reaction cross sections were fit to a functional form in Reference 2. Their ratio is shown here as a function of the pion beam energy, to indicate the strong role of pion absorption in reactions with a heavy nucleus. chain of incompletely known de-excitations. Only a large body of evidence can provide an understanding of these paths. Von Egidy provides a description of the assumed reaction chains between capture of an antiproton, also providing very large initial energy, and fission. ${ }^{19}$ A localized energy deposition, by annihilation on one nucleon for antiprotons, absorption on at least two nucleons for the pion, initiates an intranuclear cascade, followed by fast and preequilibrium emission of nucleons. At some stage, thermal equilibrium is approached, with the hot nucleus evaporating more nucleons as scission is approached, leading to fission. Further nucleon evaporation may follow fission. Since many of these steps initiated by pions may be similar to those induced by beams of photons and antiprotons, also absorbable, comparisons of fission probabilities will be made below. Comparisons between data and specific calculations following this cascade picture will be made in Section 9. This review will present all fission data available with beams of pions of both signs (which can influence the fissility parameter $Z^{2} / A$ ) over a wide range of beam energies, including the important 3-3 or $\Delta$ resonance, over a very wide range of target nuclei, from iron through plutonium.

Pions may also form an indirect pathway to fission. For instance, energetic reactions within thick samples may produce pions that in turn will induce fission elsewhere in the target, a circumstance perhaps of importance in accelerator-driven neutron sources and fission reactors. ${ }^{20}$ Virtual pions produced by high energy photons within a heavy nucleus are indicated to be of importance in photofission, ${ }^{21}$ and about five pions are created in the annihilation of an antiproton and a nucleon. If this occurs on a bound nucleon, about half of these pions will be captured to begin an intranuclear cascade of reactions which may lead to fission. ${ }^{19}$

Fission reactions from pion beams have been studied for over half a century, with initial studies using emulsion and radiochemical techniques. The emulsion studies allowed precise spatial coincidence studies, while radiochemistry can measure fission yields of a few specific masses, depending upon the radioactive decay chains. Later experiments used pulseheight analysis from electronic detectors, which enable time coincidence studies, and there is a large body of results from the use of Solid State Nuclear Track Detectors (SSNTD). The next section will present the methods used, with examples of the sensitivities of each.

1.3. Outline. Section 2 will survey the several experimental methods used to provide observables for pion-induced fission, including radiochemical, emulsion, track detector and electronic methods. Each may have its own definition of just is counted as fission, with partial information as to the masses

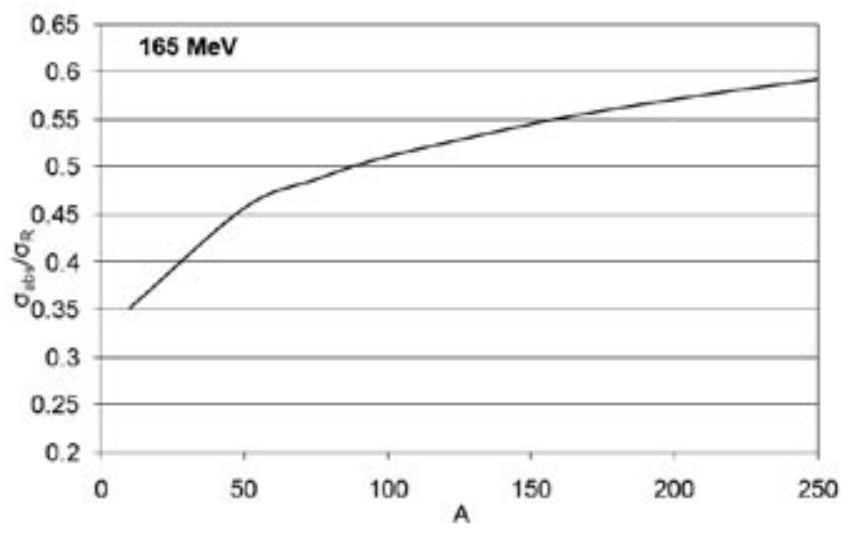

Figure 3. As in Figure 2, functional fits to absorption and reaction cross sections at the resonant energy of $165 \mathrm{MeV}$ from Reference 2 are used for the mass dependence of their ratio, with absorption of pions increasingly important for heavy nuclei. This is a reaction process important for pion-induced fission. 
and energies of the fragments studied. Taken together, a very complete range of measurements on fragment masses, fission cross sections, correlations between fragments, single fragment kinetic energies, total kinetic energies of fragments, momentum probabilities of the recoiling pre-fission nucleus, rare ternary fission cross sections, and correlations of fission fragments with lighter reaction products can be available to provide a deep view of how pions lead to fission.

Section 3 will use these methods to yield results on the mass, charge and energy distributions of fission fragments, using in many cases measurements of their ranges in material. Angular features of fission cross sections will be presented in Section 4, including fission fragment angular distributions with respect to the beam direction $\sigma_{\mathrm{f}}(\theta)$ and angular correlations between the two fragments, $\mathrm{W}(\theta)$. The target mass dependence of fission cross sections $\sigma_{\mathrm{f}}(A)$ will be presented in Section 5. There is a region from about gold $(A=197)$ to bismuth $(A=209)$ where these fission cross sections rise steeply, providing a detailed examination of the role of the pion beam charge in the fissility parameter $Z^{2} / A$. The pion beam energy dependence of fission cross sections for a few standard nuclear samples will be presented in Section 6, examing the effects of pi-nucleon resonances. A comparison to photofission will find relative differences in the resonant nature of fission induced by photons and pions. Comparisons of fission cross sections for different beams are best made by way of their probabilities, not their cross sections. These results will be shown in Section 7 , including data from stopped beams of negative pions and antiprotons.

Most of the data will have been based on two fission fragments, but rare and informative data are available for ternary fission, to be shown in Section 8. Here will also be shown the data for lighter fragments in coincidence with fission. Theoretical methods used to understand pion-induced fission will be surveyed in Section 9, with applications to the dependence of $\sigma_{\mathrm{f}}$ upon beam energy and target mass. This section also presents the reasoning behind a search for a prompt multifragmentation reaction process, and its effect upon fission cross sections. A brief set of conclusions will be found in Section 10.

\section{Experimental Methods}

2.1. Introduction. Methods to study fission induced by pi mesons have included radiochemistry, emulsion studies, SSNTD methods, and electronic methods. Each has advantages, and this section will attempt to correlate the results for a more complete understanding. SSNTD methods used several types of dielectric detector, and reported cross sections will depend also upon the SSNTD materials and the track development methods. Comparisons can provide sensitivity to fission fragment masses or energies. The many observations with SSNTD methods include some data now seen to be inconsistent with the overall trends. Some such points are not presented here, some rejected by inconsistency and some because of experimental issues noted after the runs. Many of the cross section measurements at the Los Alamos Meson Physics Facility (LAMPF) used the yields of ${ }^{11} \mathrm{C}$ to normalize the beam intensities, and thus would share the same systematic uncertainty in this quantity. This includes all measurements up to $500 \mathrm{MeV}$ from the groups in Brazil ${ }^{23-27}$ and Pakistan. ${ }^{28-32} \mathrm{~A}$ separate measurement with the Alternating Gradient Synchrotron (Brookhaven National Laboratory, USA) at higher energies used a different means to normalize the beam intensity, and included cases to connect with the LAMPF data at $500 \mathrm{MeV}^{33-36}$ These results were combined for a broad energy range in References 37-39. Other measurements used a range of techniques with less consistency among them.

2.2. Radiochemical methods. After pion bombardment, the irradiated samples may be chemically separated. With or without such separation, high resolution gamma ray spectroscopy can be used for quantitative measures of the yields of some fission products, with half lives from minutes to centuries. These data provide cross sections for specific fission product masses, and in some cases, charges, when decay chains are such as to leave one single radioisotope isolated.

The unpublished work of Russell and Turkevich, reported in Reference 40, analyzed radioactive yields from the bombardment of ${ }^{238} \mathrm{U}$ with slow negative pions, for which it is assumed that the pions are captured from rest by way of atomic orbits. Forty species were measured, with an overall symmetric spectrum of fission product masses, a sign of fission from high excitation at scission. The total fission probability, per atomic capture, was given as $0.48(0.09)$. This may be compared to electronic measurements in Section 7.

Unpublished work from the LAMPF meson factory used three energies for positive pions, 100,190 , and $350 \mathrm{MeV}$, on a uranium sample. ${ }^{41}$ Most fission products showed maximum yields at $190 \mathrm{MeV}$, following the Delta resonance. The largest yield $\left({ }^{103} \mathrm{Ru}\right)$ was $100 \mathrm{mb}$, and the sum of observed fission product radioactivities at $190 \mathrm{MeV}$ was $600 \mathrm{mb}$. The authors estimate this to be about $25 \%$ of the total fission yield. The mass yields were symmetric. Surprisingly large cross sections of 57-97 mb were found for $A=237$, produced by single-nucleon knockout reactions in competition with absorption on two nucleons and other more complex reactions, such as fission. Recent work on the systematics of pion quasifree scattering from one nucleon within a complex nucleus ${ }^{42}$ leads to an estimate of $370 \mathrm{mb}$ for the sum of such single-nucleon scattering and charge exchange total cross sections for $500 \mathrm{MeV}$ pions on uranium. This mechanism leads to a lower likelihood of fission than absorption, due to the lower energy left in the target system with quasifree scattering on only one nucleon.

Nishi et al. used radiochemical and decay methods for products from $870 \mathrm{MeV}$ negative pions on a bismuth sample. ${ }^{43}$ Their analysis emphasized spallation products, near the target mass $A=209$. Their total fission cross section for $\mathrm{Bi}$ was near $50 \mathrm{mb}$, considerably lower than observed for $1 \mathrm{GeV}$ protons on bismuth. This indication of the role of the projectile charge will be examined in more detail below in Section 3.

2.3. Emulsion methods. An early experiment exposed uranium-loaded photographic emulsion to slow $\pi$, which may stop to be captured from atomic orbits to induce fission from the rest mass of the pion. ${ }^{44}$ That work compared this pion-induced fission to that induced by fast neutrons and high energy photons, with microphotographs to show the tracks. The special features of pions and fission were first noted in this work, with distributions of fragment ranges strikingly different for slow pions and slow neutrons. It was estimated that half of pions absorbed onto uranium lead to fission, consistent with the estimate of Russell and Turkevich. ${ }^{40}$

Photographic emulsions loaded with uranium were also the subject of pion bombardments with $300 \mathrm{MeV}$ positive pions ${ }^{45}$ and $280 \mathrm{MeV}$ positive pions. ${ }^{46}$ These two analyses seem to have been based on the same experimental exposure to pions, with the sensitivities of the emulsions to tracks described by Denisenko. ${ }^{45}$ The high spatial resolution and track density resolution obtained led to spatial coincidence studies between the fission fragments and lighter reaction products. Denisenko found the single-peaked distribution of fragment ranges in emulsion to be identical for slow negative pions, $350 \mathrm{MeV}$ protons, and $300 \mathrm{MeV} \pi^{+}$, in agreement with the stopped pion data of Reference 44. While the angles between the directions of light ion fragments from proton-induced fission were isotropic, those between fragments from the pion events peaked back-toback. This is as expected if the fission had been caused by the initial absorption of the pion on two nucleons, ejected back-toback to conserve momentum. The positive pion fission cross 
section for uranium was estimated at $1000 \mathrm{mb}^{45}$

A more detailed study was presented by Ivanova, ${ }^{46}$ with events selected from among clear fission tracks as a 'trigger'. Two types of emulsion were used, one capable of recording minimum ionizing particles and the other with an ionization sensitivity above that of tracks from $45-50 \mathrm{MeV}$ protons. The background from the beam allowed only a short exposure with the more sensitive emulsion, with 73 and 460 fission events analyzed in the two cases. Both emulsion types recorded higher numbers of light charged particles per fission with positive pions than observed with $350 \mathrm{MeV}$ protons or stopped $\pi$. This is consistent with the absorption of the $\pi^{+}$onto a quasideuteron, with the emission of two protons. The more sensitive emulsion found 2.1(0.17) charged particles per fission, but that insensitive to high energy protons found $1.27(0.17)$. This limited energy analysis is consistent with the production of two energetic protons removing much of the initial reaction energy from the nuclear system. This clue will be pursued further in Section 4 using the angular information available from the work of Ivanova. ${ }^{46}$ The multiplicities of light ions associated with fission in SSNTD detectors will be presented in Section 8 .

Stars, with several heavily ionizing $(Z>4)$ particles, were found 24 and 65 times in the two emulsion exposures, with equivalent numbers of prongs, or heavily ionizing events. These nonfission events could have occurred on the $\mathrm{Ag}$ and $\mathrm{Br}$ nuclei of the emulsion, which are many times more common than uranium in the sample.

2.4. SSNTD methods. Solid State Nuclear Track Detectors are insulators which retain ionization damage from the passage of charged particles. Chemical etching attacks the damaged regions preferentially, and can reveal the tracks left. Fission fragments leave particularly dense ionization, making these SSNTD systems useful for scarce fission events, such as those from weak beams of pions and fission samples thin relative to the range of fission fragments. One can overdevelop the SSNTD to leave obvious pits, which can be counted to measure the flux of fission fragments. This method will not be sensitive to details of the SSNTD, the fission events, or the development process. Hicks et al. placed arrays of Cronar SSNTD in vacuum around nuclear samples exposed to pion beams, measuring the angular distributions of the fission fragments. ${ }^{47,48}$ This angular information will be presented in Section 4.

A more efficient means to collect fission events is to place the SSNTD in near contact with the fission sample, allowing the lightly ionizing beam to pass through both and developing

TABLE 1: SSNTD materials

\begin{tabular}{lcc}
\hline \multicolumn{1}{c}{ Material } & $Z$ & $Z / \beta$ \\
\hline CR39 & 1 & 60 \\
CN85 & 2 & 164 \\
Makrofol & $8>\mathrm{Z}>2$ & 204 \\
Cronar & 16 & 100 \\
Lexan & $16>\mathrm{Z}>8$ & 230 \\
Mica & $\mathrm{Z}>16$ & 400 \\
Phosphate glass & $\mathrm{Z}>16$ & 480 \\
Soda lime glass & $\mathrm{Z}>16$ & 620 \\
\hline
\end{tabular}

Each SSNTD material and its chemical etching provide tracks only when the ionization density is above some threshold. Here are listed the sensitivities for the SSNTD materials used for data in this review, by the charge and the ratio of the charge to ion velocity, in units of c. $^{28}$ In the SSNTD stacks of References 28-39, the plastic or glass materials were preceded by $150 \mu \mathrm{m}$ of mica, such that the succeeding materials could only register protons above $5 \mathrm{MeV}$ and alpha particles above $19 \mathrm{MeV}$. The Makrofol of References 23-27 registered fission fragments of energies with $Z>16, E>18 \mathrm{MeV}$. Cronar was used in References 47 and 48 the SSNTD lightly, so as to reveal details of the fission tracks but leave the pion tracks invisible. Since the fission fragments will not all enter perpendicular to the SSNTD, and may degrade in the sample, corrections are needed to evaluate absolute cross sections. The methods are described by de Lima et $a l .{ }^{49}$ In the samples prepared and analyzed in Pakistan the fission samples were evaporated directly onto one SSNTD, for a $2 \pi$ measurement in solid angle. ${ }^{28,30}$ In some exposures a second SSNTD was pinned to such a sample on the opposite side to maintain positioning for a $4 \pi$ measurement. Nuclear samples ranged from about 0.3 to $1.2 \mathrm{mg} / \mathrm{cm}^{2}$, with the thicknesses known to an accuracy of $7 \%$. Other work, including all results analyzed in Brazil, ${ }^{23-26,50}$ used the same fission samples about 1 $\mathrm{mg} / \mathrm{cm}^{2}$ thick mounted on thin plastic backings ${ }^{23-27}$ or actinide samples on thick stainless steel backings. ${ }^{23}$ The SSNTD sensors were separated from the sample by less than $1 \mathrm{~mm}$ of air. These studies are thus free of uncertainties of target thickness for surveys of the beam energy dependence of relative cross sections. Bismuth samples were used at both the upstream and downstream ends of the stacks, with the same cross sections measured, within uncertainties. This indicates that reactions within the stacks did not add new particles to induce fission elsewhere in the stack. Several of these samples were re-analyzed by Rutherford back scattering for improved thickness measurements. ${ }^{50}$ Cross sections reported here use these later determinations of the absolute cross sections.

Batusov et al. used mica SSNTD to sense fission fragments from the capture of stopped $\pi^{-}$on 15 nuclei, from Ag to U. ${ }^{51}$ Khan et al. used several types of SSNTD, with a range of thresholds for $Z$ and $Z / \beta$ ( $\beta$ as the ratio of the ion speed to that of light) to enable separation of fission events from other processes. $^{28}$ Table 1 lists the SSNTD types used in that work, and adds references to the materials used by other fission studies with SSNTD. Several of the SSNTD pion-fission experiments were calibrated with spontaneous fission sources, with SSNTD materials and development exactly as used with the beam exposures. Examples of track length comparisons will be shown in Section 3.

Yasin measured fission cross sections for $\pi^{-}$at kinetic energies of 500, 672, and $1068 \mathrm{MeV}$ for Bi with three types of SSNTD materials, each with its appropriate etching methods. ${ }^{35}$ The cross sections obtained with Makrofol, CR39 and mica are shown in Figure 4. The average cross sections are 99, 102, and $144 \mathrm{mb}$ at 500,672 , and $1068 \mathrm{MeV}$ respectively, with the standard deviation of the mean for each energy averaging $11 \%$. We may take this as the systematic uncertainty in $2 \pi$ SSNTD abso-

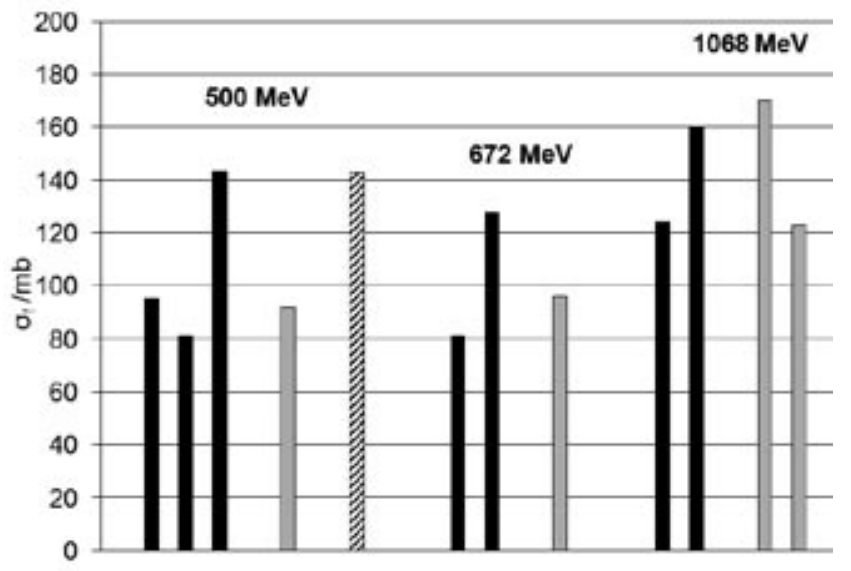

Figure 4. Fission cross sections for bismuth at three energies were measured with three types of SSNTD. ${ }^{36}$ Here are shown the inferred cross sections, with solid bars for Makrofol, open bars for CR39, and cross hatching for mica. The scatter among these results at each energy is used to establish a systematic uncertainty of $11 \%$ for this method. 
lute cross sections due to the choice of detector material and uncertainties in sample thicknesses in the Pakistani method.

Some SSNTD studies used $4 \pi$ geometry, able to correlate forward and backward going fission fragments, using close control of the orientation of material downstream and upstream from the fission sample. This enables the determination of the angular correlation $\mathrm{W}(\theta)$ between the two fission fragments, as presented in Section 4. Other experiments used $2 \pi$ geometry, catching only one of the fragments, usually downstream from the fission sample relative to the pion beam direction. This is the most efficient means to determine fission cross sections, not using the details of track ionization and track angle. Ternary fission events have been counted as three tracks in the $4 \pi$ geometry, or from two tracks, not back-to-back, in $2 \pi$ geometry. ${ }^{28}$

Development of the SSNTD can be calibrated so as to make observable the angles of the tracks relative to the surface of the SSNTD material. ${ }^{28}$ These observations allow determination of the angular distributions of the fission fragments relative to the beam direction, a method complementary to the method of Hicks. ${ }^{47,48}$ The results will be compared in Section 4. The development system used in Reference 28 also makes tracks from lighter fragments, such as protons, observable, also with angular information. Because of the lengths of these tracks, exact angular correlations with fission tracks are not possible, in distinction to the use of emulsions, but tracks were only counted if associated with fission tracks. Cross sections for these light reaction products and their angular distributions will be shown in Section 8 .

Exposures of SSNTD were carried out with $500 \mathrm{MeV} \pi^{-}$at two facilities, LAMPF and the AGS. The LAMPF method used Makrofol and the standard set of targets, ${ }^{26}$ while the AGS exposure used several types of SSNTD, each with its own evaporated nuclear sample. ${ }^{31,35,36}$ These two sets of exposures also used very different means to monitor the pion beam fluence. With four measurements, the average $500 \mathrm{MeV} \pi$ fission cross section for bismuth was $88.5 \mathrm{mb}$, with an uncertainty from the scatter among the measurements of $2.7 \mathrm{mb}$. Individual measurements cited uncertainties of $14 \mathrm{mb}$. The agreement between the two exposures is thus very good. A $500 \mathrm{MeV}$ datum for tin in Reference 33 was replaced by a better measurement in Reference 34.

2.5. Electronic methods. Only two experiments have used pulse height detectors for studies of pion-induced fission. ${ }^{48,52}$ The arrays of silicon surface barrier detectors gave pulse height information for coincident pairs of fission fragments, providing the total kinetic energy (TKE) release for each event. Calibration, vital because of the pulse height defect for heavily ionizing events, was provided using a spontaneous fission source. Relative pulse heights for the two coincident fragments were sufficient to determine the mass ratio, the mass of fragment M1 divided by the sum of masses for the two fragments $M 1+M 2$. These energy and mass results will be shown, together with other determinations, in Section 3.

The angular correlation $\mathrm{W}(\theta)$ between the two coincident fragments was also determined in the electronic work of Hicks et al. $^{48}$ Results will be compared to other methods for the same fission targets at similar energies in Section 4. These electronic methods were carried out in a beam geometry that did not permit the determination of absolute cross sections or angular distributions $\mathrm{d} \sigma / \mathrm{d} \Omega(\theta)$.

\section{Track Lengths, Energies, and Masses}

3.1. Introduction. Some of the experimental methods described in Section 2 enabled determination of the energy and mass distributions of fission products. Each method was based on assumptions, so these results require careful comparisons. An observable related to both of these variables is found in the

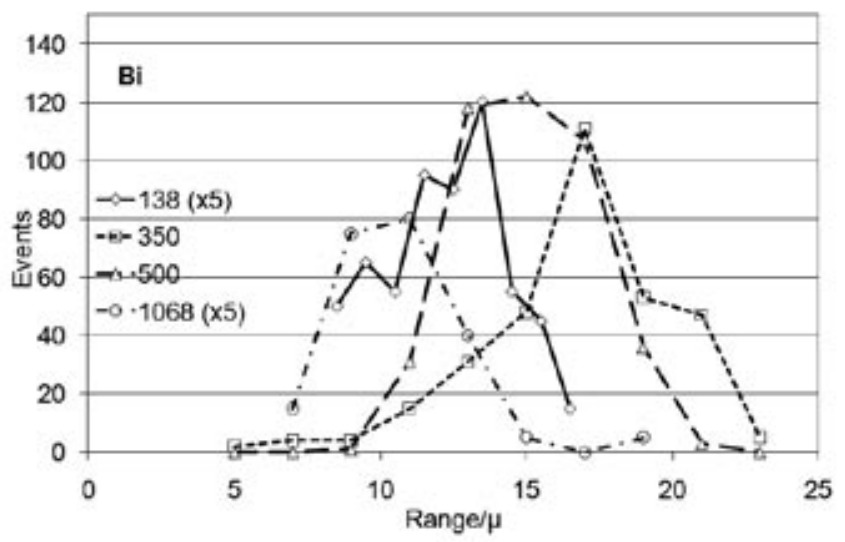

Figure 5. Distributions of track lengths measured for events from negative pion bombardment of bismuth are shown, measured with plastic SSNTD. Event numbers for $138 \mathrm{MeV}^{23}$ and $1068 \mathrm{MeV}$ negative pions ${ }^{33}$ have been multiplied by five. The $350 \mathrm{MeV}$ and $500 \mathrm{MeV}$ $\pi^{-}$data are from the Brazilian group. ${ }^{50}$

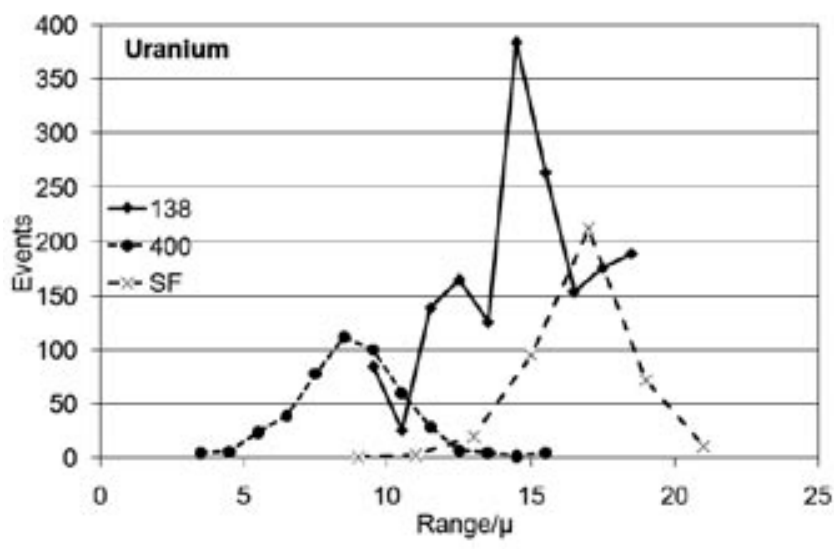

Figure 6. As Figure 5, but for a uranium sample and positive pions The shorter ranges at $400 \mathrm{MeV}$ are in a mica sample. ${ }^{53}$ Also shown are track lengths in Makrofol from a spontaneous fission (SF) calibration source of ${ }^{242} \mathrm{Pu}{ }^{50}$

ranges of fission tracks, in emulsion or in dielectric SSNTD. With a given SSNTD material, track length registrations for different fragment energies or masses depend upon thresholds for the charge and charge to velocity of the resulting fission fragments. In one case, identical SSNTD methods were used for fission induced by pions, protons or neutrons, so the pion results can be placed into more familiar contexts with wider ranges of experimental results.

3.2. Track lengths. The most fundamental measurement from fission events sensed in SSNTD is the track length distribution of fragments in the material, determined by the energy, charge, and mass of the fragment. A first question is whether these ranges for a given target depend upon the beam energy of the pion. Four $\pi^{-}$energies for a bismuth sample yield the range distributions shown in Figure 5, while $\pi^{+}$data for a uranium sample are shown in Figure 6. Observations of tracks in Makrofol from a ${ }^{242} \mathrm{Pu}$ spontaneous fission (SF) source are also shown. Since these data come from several sources, it is not useful to compare their average values to seek trends.

A second study of interest would be the distribution of track lengths at a fixed energy for several targets. Examples have been shown in References 23, 26, 39, and 53. Figure 7 shows track length distributions for events induced in a mica sandwich by $400 \mathrm{MeV} \pi^{+}$for uranium and gold, with results also for events induced in uranium by $14 \mathrm{MeV}$ neutrons. ${ }^{53}$ These data are from SSNTD both downstream and upstream of the nuclear samples, so that momentum imparted by the beam might be noted. The pion beam momentum is $522 \mathrm{MeV} / \mathrm{c}$, and that for 


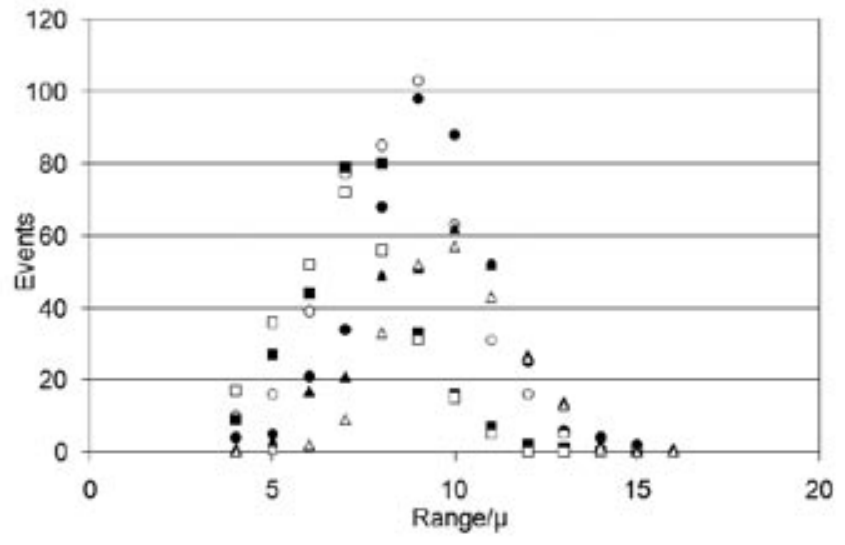

Figure 7. The momentum of the pion beam may be noted in the number and the ranges of tracks observed up-stream (open) and down-stream (solid) of the sample. Here are shown fission fragment track lengths in mica $^{53}$ for $400 \mathrm{MeV} \pi^{+}$incident on $\mathrm{UF}_{4}$ (circles) and $\mathrm{Au}$ (square). For comparison, tracks from events due to $14 \mathrm{MeV}$ neutrons are also shown (triangles), using the same methods. ${ }^{53}$

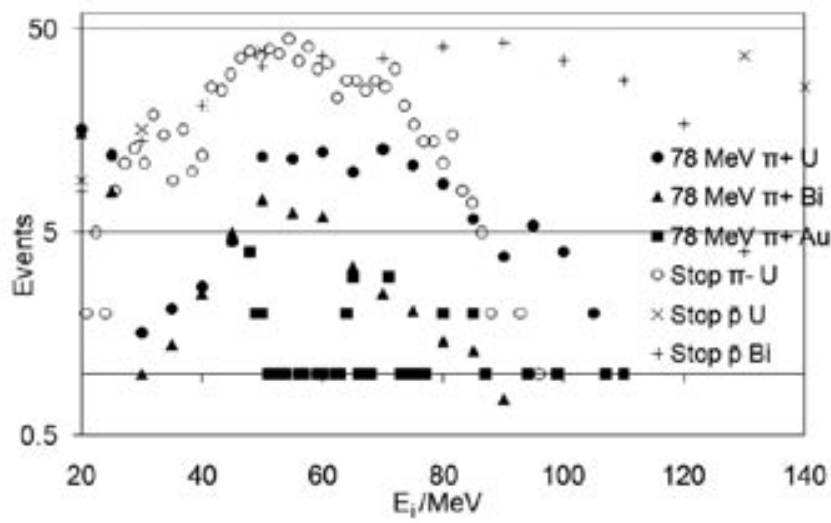

Figure 8. The kinetic energies of single fission fragments were measured in silicon counters for $78 \mathrm{MeV} \pi^{+}$incident upon samples of uranium (solid circle), bismuth (solid triangle), and gold (solid square) ${ }^{45,46}$ and for $\pi$ stopped in ${ }^{235} \mathrm{U}$ (open circles). ${ }^{52}$ Also shown are measurements from the fission of uranium (x) and bismuth (+) by stopped antiprotons. ${ }^{56}$

$14 \mathrm{MeV}$ neutrons is $163 \mathrm{MeV} / \mathrm{c}$. Average track lengths downstream/upstream are 9.17/8.45 $\mu \mathrm{m}$ for $U$ and 7.57/7.26 $\mu \mathrm{m}$ for $\mathrm{Au}$ with pions, and 9.49/9.36 $\mu \mathrm{m}$ for $\mathrm{U}$ with neutrons. ${ }^{53}$ Some of the pion beam momentum is found in longer forward tracks, longer for uranium than for gold because of the larger charge at scission. The downstream/upstream ratio is less for the neutrons, with less momentum, and the longer tracks may be due to the loss of less charge before scission than was the case with pions.

3.3. Single event energies. Surface barrier silicon counters were used to measure the energies of single fission events $E_{\mathrm{i}}$ for stopped $\pi^{-}$in ${ }^{235} \mathrm{U}^{54}$ and for $78 \mathrm{MeV} \pi^{+}$on uranium and bismut, ${ }^{48}$ as shown in Figure 8. Sparse data for $78 \mathrm{MeV} \pi^{+}$on gold are also shown. ${ }^{47}$ These events were registered in time coincidence with a second detector, and are clearly due to fission. ${ }^{48,54}$ The average single fragment energy above $25 \mathrm{MeV}$ is measured from these data to be $68 \mathrm{MeV}$ for the $\pi^{+}$and $58 \mathrm{MeV}$ for the energetic $\pi^{-}$. The average single fragment energy for stopped $\pi^{-}$is $57 \mathrm{MeV}{ }^{54}$ These comparisons may indicate some degree of retention of the beam charge leading to fission.

3.4. Total kinetic energies. Only one experiment at low pion energies presented the sum of two fission fragment energies (TKE) by calibrated and summed pulse heights. ${ }^{48}$ Pulse heights were calibrated using a ${ }^{252} \mathrm{Cf}$ spontaneous fission source; this calibration includes the pulse height defect, for the loss of pulse height by recombination of the ions in the dense

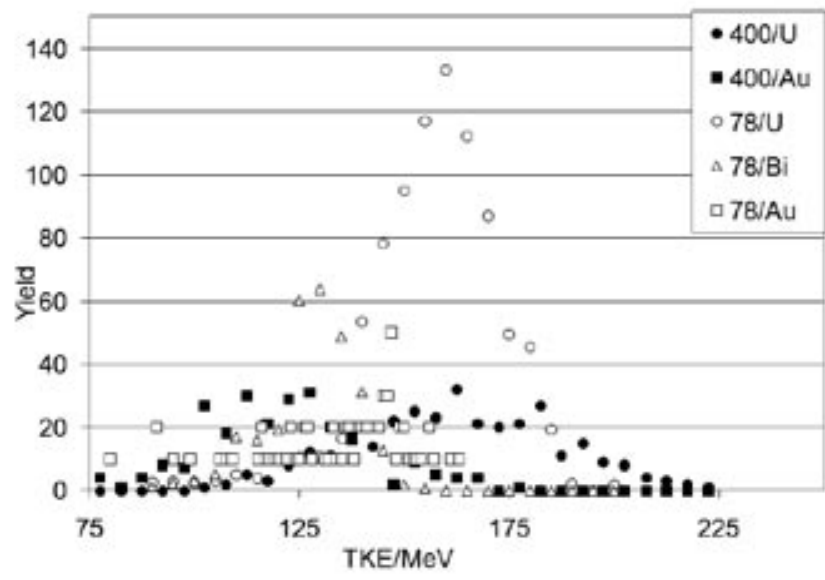

Figure 9. The sum of kinetic energies (TKE) of two coincident fission events in silicon counters ${ }^{48}$ is shown for $78 \mathrm{MeV} \pi^{+}$incident (open points) upon samples of $\mathrm{U}, \mathrm{Bi}$, and $\mathrm{Au}$. Averages are 165 and $143 \mathrm{MeV}$ for $\mathrm{U}$ and $\mathrm{Au}$, respectively. Carefully calibrated track details in a 'mica sandwich' from the $400 \mathrm{MeV} \pi^{+}$bombardment of $\mathrm{U}$ and $\mathrm{Au}$ samples were analyzed to give the TKE distributions shown here as solid points, for $\mathrm{U}$ and $\mathrm{Au}$, with averages of 162(15) and 123(9) $\mathrm{MeV}$ for $\mathrm{U}$ and $\mathrm{Au}^{53}$

TABLE 2: Average track lengths

\begin{tabular}{cclcllc}
\hline Sample & Beam & Material & $\langle\mathrm{L}\rangle$ & $+/-$ & $\sigma$ & Reference \\
\hline $\mathrm{Sn}$ & -500 & Makrofol & 8.7 & 0.17 & 1.92 & 26 \\
$\mathrm{Au}$ & +400 & Mica* & 7.26 & 0.10 & 1.76 & 53 \\
& +400 & Mica & 7.57 & 0.092 & 1.59 & 53 \\
$\mathrm{Bi}$ & -138 & Makrofol & 12.2 & 0.2 & 2.1 & 23 \\
& -350 & Makrofol* & 12.85 & 0.22 & 2.44 & 50 \\
& -350 & Makrofol & 16.7 & 0.18 & 3.2 & 50 \\
& -500 & Makrofol & 15.0 & 0.11 & 2.26 & 50 \\
& -500 & Makrofol & 13.6 & 0.2 & 1.78 & 26 \\
& -1068 & CR39 & 10.8 & 0.3 & 2.2 & 33 \\
${ }^{235} \mathrm{U}$ & +138 & Makrofol & 14.8 & 0.1 & 1.82 & 23 \\
$\mathrm{U}$ & +400 & Mica* & 8.45 & 0.09 & 1.90 & 53 \\
$\mathrm{U}$ & +400 & Mica & 9.17 & 0.09 & 1.90 & 53 \\
$\mathrm{U}$ & -500 & Makrofol & 12.47 & 0.3 & 2.73 & 26 \\
${ }^{242} \mathrm{Pu}$ & $\mathrm{SF}$ & Makrofol & 17.6 & 0.13 & 2.16 & 50 \\
${ }^{242} \mathrm{Pu}$ & $\mathrm{SF}$ & Makrofol & 16.7 & 0.1 & 1.79 & 23 \\
\hline
\end{tabular}

Average track lengths $<\mathrm{L}>$ and their uncertainties are given for pioninduced fission events in SSNTD, for a range of nuclei and pion beam energies. The width $\sigma$ of a presumed Gaussian distribution is also given. All lengths are in micrometers $(\mu \mathrm{m})$. All samples were downstream of the target except for those starred, which were upstream. Widths and uncertainties from the work of Dautet ${ }^{53}$ were measured for the present work. Distributions of track lengths are shown in Figure 5. Calibrations with spontaneous fission (SF) are also listed.

fragment track. Figure 9 shows the TKE for $78 \mathrm{MeV} \pi^{+}$on $\mathrm{U}$ and $\mathrm{Bi}$, with higher TKE from uranium as expected from its greater charge.

Analysis and careful calibration of track lengths has also been used to infer the energies of correlated pairs of fission fragments in a 'mica sandwich' for $400 \mathrm{MeV} \pi^{+} .53$ These distributions are compared to the electronic results in Figure 9, with both studies showing data for a uranium sample. The fitted average TKE for uranium are $164.9(0.3) \mathrm{MeV}$ at $78 \mathrm{MeV}$ and $162(15) \mathrm{MeV}$ at $400 \mathrm{MeV}$. Evidently the $\pi^{+}$beam energy, available as part of the initial nuclear energy budget, has little effect of the average TKE. Table 3 lists fitting results for these and other data. The results at $400 \mathrm{MeV}$ give greater widths for the TKE distributions than is noted for the lower energy electronic data. It is not clear whether this is an artifact of the mica 
sandwich method or a true effect of the higher beam energy.

Higher energy pions bring higher grazing angular momentum, and if this angular momentum were to remain to fission, the average TKE is expected to increase. ${ }^{1}$ In fact, the uranium $\pi^{+}$TKE data at $65,78,96,{ }^{48}$ and $400 \mathrm{MeV}^{53}$ remain constant, in spite of the increase in the grazing angular momentum, from 6 to 21 units. The angular momentum carried by the pion beams seems to have little influence on the fission dynamics. Identical measurements of TKE for $U$ with a mica sandwich were carried out with $400 \mathrm{MeV} \pi^{+}, 14 \mathrm{MeV}$ neutrons, and thermal neutrons. ${ }^{53}$ It is presumed that the thermal neutron fission of natural uranium in that work is due to the ${ }^{235} \mathrm{U}$ isotope only. As shown in Table 3, the pion TKE are lower than for neutrons.

Average TKE values for $\pi^{+}$are plotted in Figure 10 as in Fig. $\mathrm{X}-1$ of Reference 1 , against the variable $Z^{2} / A^{1 / 3}$ for measurements at 65,78 , and $95 \mathrm{MeV}^{48}$ and at $400 \mathrm{MeV} .^{53}$ If the TKE is derived from the Coulomb repulsion of two fragments, this argument should be the relevant one for a linear plot. This plot of pion data uses $A$ as the mass of the target nucleus, and $Z$ to include the beam pion charge and the target nuclear charge. The line shows the equation of Reference 55, $<$ TKE $>=0.1071$ $Z^{2} / A^{1 / 3}+22.2 \mathrm{MeV}$, also shown in Reference 1 to represent a wide range of data. The pion points lie below this trend, with these assumptions. If the expression is used instead to infer the charge of the fissioning system, assuming a loss of an average of three nucleons from $A$, the $78 \mathrm{MeV}$ average TKE values indicate that the charge of the target drops by two or three for $\mathrm{Bi}$ for both signs; the $400 \mathrm{MeV} \pi^{+}$results agree with this change. A change for Au of two mass units for $\pi^{-}$, none for $\pi^{+}$ is indicated at $78 \mathrm{MeV}$, with a loss of about six charges for $\pi^{+}$at $400 \mathrm{MeV}$. These fission TKE values are giving useful clues as to the processes preceding pion-induced fission.

3.5. Fission fragment masses. Radiochemical methods gave both the fragment mass and energy dependence of barium isotope fission fragments for $\pi^{+}$beams on uranium. ${ }^{41}$ Data for five barium isotopes allowed a study of the role of fission product neutron excess. ${ }^{41}$ Cross sections at three pion beam energies are shown in Figure 11. Neutron-rich isotopes (relative to $A=137.3$ for stable barium) show the resonance structure, but the lower yields for lighter isotopes increase with beam energy. This may indicate that the higher beam energies gave greater nucleon emission before fission. The authors also state that the ratio of ${ }^{131} \mathrm{Ba} /{ }^{140} \mathrm{Ba}$ yields for pions is near that for proton-

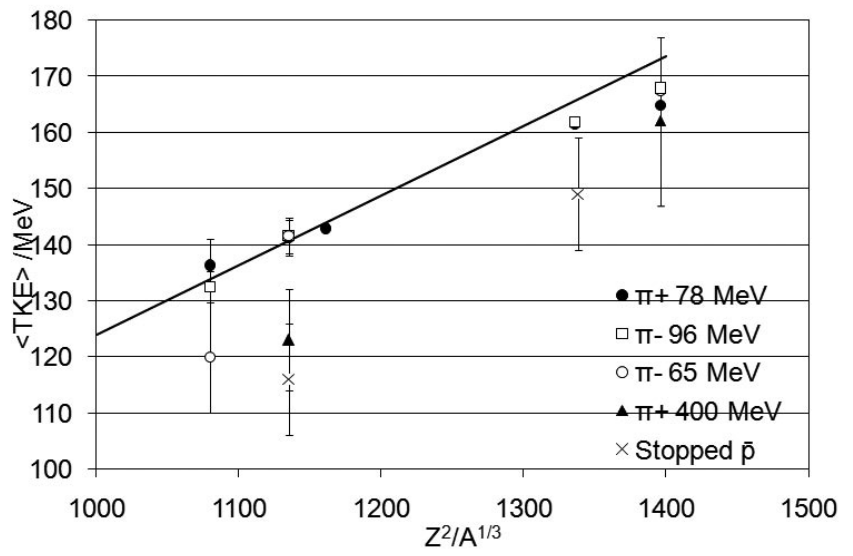

Figure 10. Total kinetic energy averages for 78 and $96 \mathrm{MeV}$ pions of both signs, ${ }^{48} 400 \mathrm{MeV}$ positive pions ${ }^{53}$ and stopped negative pions ${ }^{58}$ are plotted against a parameter $Z^{2} / A^{1 / 3}$, with the assumption that the charge $Z$ of the fissioning system is that of the target nucleus plus the pion beam charge, and $A$ is the mass of the target nucleus. The line shows the relation $\left\langle\mathrm{TKE}>=0.1071 Z^{2} / A^{1 / 3}+22.2 \mathrm{MeV}\right.$, as compared to a wide range of data in Figure $\mathrm{X}-1$ of Reference 1 . This is the expression used for the discussions in the text.

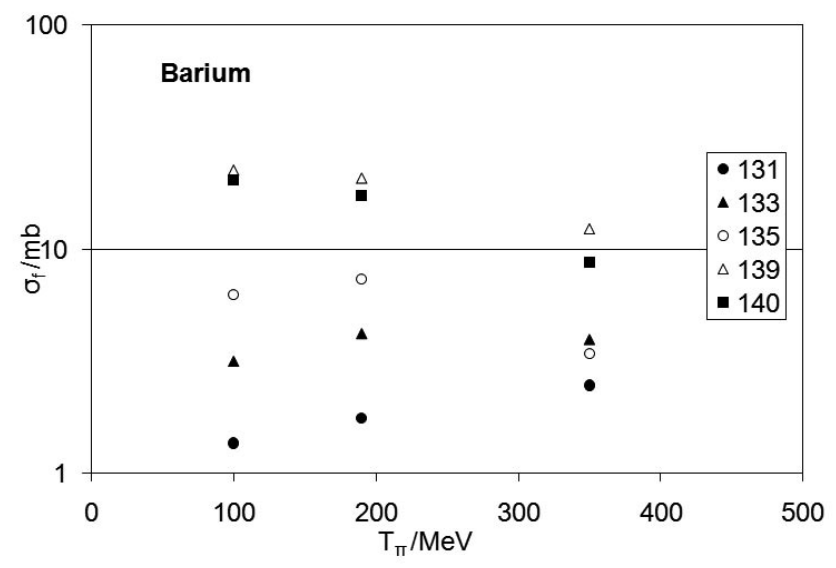

Figure 11. Radiochemical techniques provided the mass yields shown for isotopes of barium, resulting from 100,190 and $350 \mathrm{MeV}$ $\pi^{+}$incident on uranium. ${ }^{41}$

TABLE 3: Energy and mass distributions

\begin{tabular}{|c|c|c|c|c|c|c|c|}
\hline Sample & Pion energy & $<\mathrm{TKE}>$ & FWHM & $<\mathrm{M}>$ & FWHM & $<\mathrm{Ei}>$ & FWHM \\
\hline $\mathrm{Au}$ & +78 & $141.2(3.1)$ & $21.6(3.0)$ & $96(6)$ & $46.5(3.3)$ & - & - \\
\hline $\mathrm{Au}$ & -78 & $136.3(4.7)$ & - & - & - & - & - \\
\hline $\mathrm{Au}$ & +96 & $141.8(0.9)$ & $24.8(1.5)$ & $99(3)$ & $48.7(2.8)$ & - & - \\
\hline $\mathrm{Au}$ & +400 & $123(9)$ & 42 & $95.0(2.5)$ & 55 & - & - \\
\hline $\mathrm{Bi}$ & +78 & $142.9(0.3)$ & $23.6(0.6)$ & $105(5)$ & $60.6(1.5)$ & 56.6 & $27(4)$ \\
\hline $\mathrm{Bi}$ & p-bar or anti-proton & $116(10)$ & - & $94(2)$ & 54 & 76 & 78 \\
\hline $\mathrm{U}$ & stopped pi & - & - & - & - & 57.8 & 35 \\
\hline $\mathrm{U}$ & -38 & - & - & 119 & 61 & - & - \\
\hline $\mathrm{U}$ & +78 & $164.9(0.3)$ & $29.3(0.5)$ & 120 & $66.2(1.2)$ & 67.8 & $45(5)$ \\
\hline $\mathrm{U}$ & -78 & $161.4(0.2)$ & $27.2(0.3)$ & 118 & $66.2(1.9)$ & - & - \\
\hline $\mathrm{U}$ & +400 & $162(15)$ & 56 & $117.0(2.5)$ & 61 & - & - \\
\hline $\mathrm{U}$ & $14 \mathrm{MeV} \mathrm{n}$ & 178 & 65 & - & - & - & - \\
\hline $\mathrm{U}$ & thermal n & 171(10) & 75 & - & - & - & - \\
\hline $\mathrm{U}$ & p-bar or anti-proton & 149(10) & - & $106(2)$ & 42 & 88 & 71 \\
\hline
\end{tabular}

The results of fitting or measuring averages $<$ TKE $>$ and full widths at half maximum (FWHM) of distributions of TKE and mass and energy distributions of single fission fragments from pion beams are listed. The fitted values for 65 and $96 \mathrm{MeV}$ are very similar to those at $78 \mathrm{MeV}$. ${ }^{48}$ Averages for mass distributions $<\mathrm{M}>$ and their widths were measured from figures in Reference 48 for the present work. The $400 \mathrm{MeV}$ pion and the $14 \mathrm{MeV}$ neutron fits are from Reference 53, using a natural uranium target. It is presumed that fission induced by thermal neutrons is only from the ${ }^{235} \mathrm{U}$ isotope. Some data for $38 \mathrm{MeV}$ negative pions, ${ }^{52}$ stopped negative pions, ${ }^{54}$ and stopped antiprotons ${ }^{58}$ were measured from published figures for the present work. Energies are in $\mathrm{MeV}$ and masses in amu. 
induced fission at beam energies about $150 \mathrm{MeV}$ higher, as another indication of the role of absorption of the pion rest mass of $140 \mathrm{MeV}$.

No other method yields mass resolution as good as this. While the methods described next give smooth distributions of fragment masses, averaged over the resolution of the method, these radiochemical results are accurate to within one unit of $A$, and thus show greater scatter. The larger neutron excess of uranium is the basis of the larger cross sections for heavier barium isotopes. In a related work, the spallation products from 500 and $1570 \mathrm{MeV}$ pion bombardment of copper were determined by radiochemistry. ${ }^{56}$ Fission cross sections for copper at lower energies will be presented in Section 5.

Two experiments ${ }^{48,52}$ used energy signals E1 and E2 in time coincident silicon detectors to infer mass ratios of the two fission fragments by $\mathrm{M} 1 /(\mathrm{M} 1+\mathrm{M} 2)=\mathrm{E} 2 /(\mathrm{E} 1+\mathrm{E} 2)$. Results for uranium, as fractions of the total number of events, are shown in Figure 12 as a function of M1 with M1+M2 taken to be the mass of the initial target nucleus. All yields in this figure have been normalized to the magnitude of the $38 \mathrm{MeV} \pi^{-}$data. ${ }^{52}$ Figure 3 of Reference 48 shows a familiar bimodal distribution of masses from a ${ }^{252} \mathrm{Cf}$ spontaneous fission source, but all pioninduced fission mass distributions are symmetric. The full widths at half maximum (FWHM) for pions of both signs at 65,78 , and $96 \mathrm{MeV}$ average $65.3 \mathrm{amu}$ for uranium. The FWHM of the mass distribution for Au is only $47.6 \mathrm{amu}$, with fewer data. ${ }^{48}$ The full width at half maximum for the $38 \mathrm{MeV}$ data on uranium ${ }^{52}$ is measured from their data to be $61 \mathrm{MeV}$, much the same for ${ }^{238} \mathrm{U}$ and ${ }^{237} \mathrm{~Np}$. These determinations are based on the strongest assumptions and the best resolution of the methods used for pion-induced fission, and are the test by which other results can be tested. Good consistency in the mass ratio is found for uranium over a range of beam energies with both pion beam signs.

Figure 12 also shows SSNTD mass distributions for $80 \mathrm{MeV}$ pions of both signs for uranium, ${ }^{28}$ using measurements of track density for this determination. Results are very similar to the electronic data, using very different methods. Figure 4 of Reference 28 shows that the use of two different SSNTD materials gave the same mass distribution data for fission. We conclude that the SSNTD methods used by Khan et al. yield reliable fission fragment mass distributions.

Based on this confidence, we compare the fragment mass distributions from SSNTD exposures for $400 \mathrm{MeV} \pi^{+}$on $\mathrm{Au}$ and $\mathrm{U}^{53}$ in Figure 12 to the lower energy results. ${ }^{48}$ The average of the masses shown for the $400 \mathrm{MeV}$ experiment are 117.6(2.5) for $\mathrm{U}$ and 95.0 (2.5) for $\mathrm{Au}$, while the $78 \mathrm{MeV}$ averages for $\mathrm{U}$ are 120 for $\pi^{+}$and 118 for $\pi^{-}$. It appears that the pion beam energy has little effect on the average of fission fragment masses for the uranium sample. A Gaussian curve with an average $\mathrm{M}=119$ and a FWHM of 66.2 is shown for reference in Figure 12.

Hicks gives a discussion of the nuclear excitation energy carried through to fission, analyzing the widths of the energy and mass distributions. ${ }^{48}$ The conclusion was that much of the rest mass of the pion is retained at the energies of that experiment. If this were to be true also at higher beam energies we could expect strong changes in observables such as the fragment mass distribution; no such strong effects are seen in the data. More recent theoretical methods indicate that little of the initial energy is held to fission.

For a few fragment masses, radiochemical methods have given the probabilities for uranium as shown in Figure 12, not normalized to the data as the other cases. Results for $\pi^{-}$at 870 $\mathrm{MeV}$ on bismuth found the strongest yields for masses from spallation, not fission. ${ }^{43}$

The angular momentum retained by a nucleus after a $\pi^{-}$is captured from rest has been noted by the gamma ray de-excitation from rotational bands in those products in Reference 57.

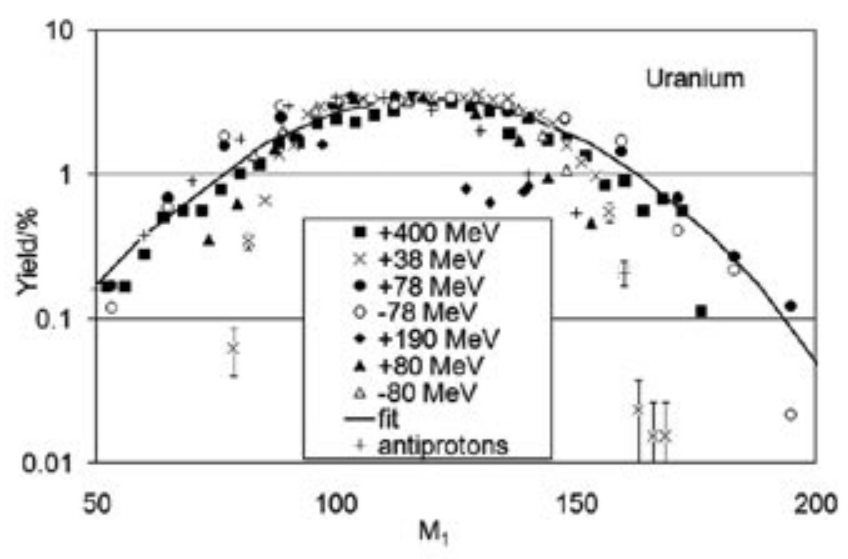

Figure 12. Mass distributions for single fission fragments have been inferred from the deposited energies ${ }^{48,52}$ or from details of track lengths and densities, ${ }^{28,53}$ and are shown for uranium. A few samples of results from $190 \mathrm{MeV} \pi^{+}$radiochemical methods ${ }^{41}$ are also shown. A curve shows a fit to the data for $78 \mathrm{MeV} \pi^{+} .{ }^{48}$ Data for antiprotons stopped in $U$ are also shown. ${ }^{58}$ All probabilities have been normalized to the results of Reference 52 .

On samples of $\mathrm{Hg}, \mathrm{Au}$ and $\mathrm{Pt}$, that work found the reaction yields to be dominated by $\left(\pi^{-}, x \mathrm{n}\right)$ reactions, with up to 16 neutrons removed. The angular momentum of the final states included population of spins as high as eleven, even for capture of stopped pions with no initial beam momentum. Emission of charged particles is, in general, much less, and the nuclei used in Reference 57 will be shown in Section 5 to have low probabilities for fission. Fission products were not reported for these nuclei in that work.

3.6. Mass and energy comparisons. Fission experiments with stopped ${ }^{54}$ and energetic ${ }^{48,52,53}$ pions and with stopped antiprotons ${ }^{58}$ have presented figures or fitted values for TKE of fission fragments, the masses of single fragments $\left(M_{\mathrm{i}}\right)$, and the energies $\left(E_{\mathrm{i}}\right)$ of single fragments. Some of the averages and widths of these distributions have been measured from published figures for the entries in Table 3, to compare trends with target mass, with pion beam energy, and with two beam charges. Data for fission of uranium induced by thermal and $14 \mathrm{MeV}$ neutrons are also presented for comparison. ${ }^{53}$ Since methods used differ among these data sets, comparisons can only be general.

Kinetic energy distributions $E_{\mathrm{i}}$ of single fission fragments for energetic positive pions ${ }^{48}$ and for stopped pions ${ }^{54}$ and antiprotons ${ }^{58}$ are compared in Figure 9. The widths of the antiproton distributions were measured for the present work. The average $E_{\mathrm{i}}$ and the widths of the distributions increase with the initial energy available, based on very few comparisons.

Average TKE for fission fragments for several beams are also listed in Table 3. For both Bi and U nuclei, these averages are lower for fission induced by stopped antiprotons than for pions. If the antiproton reaction with its greater available energy removed more charge than the pion reactions before fission, this would be the expected effect. The widths of the pion TKE distributions seem to increase with beam energy, but are narrower than the distributions noted with neutrons.

Inferred masses of single fission fragments for stopped antiprotons and pions and for energetic pion beams are compared in Figure 12, and average values are compared in Table 3. For uranium there is a clear trend for the average mass to decrease for reactions with more initial energy. There is less evidence of this for bismuth. The width of the mass distributions, a measure of the excitation energy at scission, is less for stopped antiprotons than for the pion cases for $\mathrm{Bi}$, but greater for $\mathrm{U}$. 


\section{Angular Information}

4.1. Introduction. The angular distribution of fission fragments, relative to the beam direction, is a measure of the projection of the nuclear angular momentum on the symmetry axis. ${ }^{1}$ Since the light pions bring in little angular momentum (less than 6 units for a $80 \mathrm{MeV}$ pion at grazing incidence on uranium), these angular distributions $\mathrm{d} \sigma / \mathrm{d} \Omega(\theta)$ are expected to be nearly isotropic. Two experiments using very different methods have determined these distributions. Determinations of the angular correlation $\mathrm{W}(\theta)$ between two binary fission fragments specify the momentum of the recoiling system at scission, since in their center of mass frame those fragments will be back-to-back. The momentum from the beam carries the initial system forward, but processes before fission can remove some of this full linear momentum transfer (FLMT). These early processes remove energy, and observations of the width of the angular correlation patterns can be used to infer the excitation energy of the system at scission. ${ }^{48}$ The more violent multifragmentation reaction can also produce fragments, but the angular spread of the two heaviest fragments from this process is expected to be much wider apart from back-toback. ${ }^{59}$ The electronic methods of Hicks et al. ${ }^{48}$ and the detailed track angle studies with SSNTD ${ }^{28}$ can determine these correlations, while simpler downstream-to-upstream ratios can be used for an estimate of the momentum of the recoiling system. The good spatial and track density information from emulsion studies is able to give angular correlation information between the light particles in spatial coincidence with fission, presumably from fast processes before scission. These results confirm the important role of pion absorption inducing fission. Less precise SSNTD methods have given the angular distribution of single light fragments in association with fission. This section will present and compare these results of angular measurements.

4.2. Angular distributions. The most accurate angular distribution data are those of Hicks et al., using over-developed Cronar SSNTD in an array around the nuclear sample. ${ }^{48}$ Figure 13 shows uranium data at $80 \mathrm{MeV}$, fit to $\mathrm{A}+\mathrm{B} \cos ^{2}(\theta)$. Fitted coefficients are listed in Table 4. As expected, the fragments

TABLE 4: Angular distributions

\begin{tabular}{ccccc}
\hline Beam & Sample & $\mathrm{A}(\mathrm{mb} / \mathrm{sr})$ & $\mathrm{B}$ & $\sigma_{\mathrm{f}}(\mathrm{mb})$ \\
\hline+60 & $\mathrm{U}$ & $262(26)$ & 0.070 & $1680(170)$ \\
+70 & $\mathrm{U}$ & $308(31)$ & 0.049 & $1970(200)$ \\
+80 & $\mathrm{U}$ & $305(31)$ & 0.063 & $1960(200)$ \\
+90 & $\mathrm{U}$ & $305(31)$ & 0.049 & $1940(190)$ \\
+100 & $\mathrm{U}$ & $337(34)$ & 0.041 & $2140(210)$ \\
-60 & $\mathrm{U}$ & $453(57)$ & 0.113 & $2960(370)$ \\
-70 & $\mathrm{U}$ & $470(47)$ & 0.049 & $3000(3000$ \\
-80 & $\mathrm{U}$ & $434(43)$ & 0.051 & $2770(280)$ \\
-90 & $\mathrm{U}$ & $349(35)$ & 0.083 & $2260(2300)$ \\
-100 & $\mathrm{U}$ & $366(37)$ & 0.090 & $2360(240)$ \\
+80 & $\mathrm{Bi}$ & $43.8(4.4)$ & 0.001 & $276(28)$ \\
-80 & $\mathrm{Bi}$ & $14.7(1.5)$ & 0.096 & $100(10)$ \\
+80 & $\mathrm{U}$ & $309(7)$ & 0.124 & $2020(45)$ \\
+80 & $\mathrm{~Pb}$ & $38.6(2.5)$ & 0.216 & $260(17)$ \\
+80 & $\mathrm{Au}$ & $11.3(1.3)$ & 0.327 & $79(9)$ \\
+80 & $\mathrm{Ho}$ & $0.43(0.29)$ & $0.33(0.13)$ & $3(2)$ \\
\hline
\end{tabular}

The fitted coefficients $\mathrm{A}$ and $\mathrm{B}$ for the angular distributions $\mathrm{A}+\mathrm{B} \cos ^{2}(\theta)$ of pion-induced fission are listed for a range of beam energies (in $\mathrm{MeV}$ ) of both signs on uranium and bismuth ${ }^{48}$, and for 80 $\mathrm{MeV}$ positive beams for a range of nuclei. ${ }^{28}$ The total fission cross sections in mb are also given. Good agreement is found from the very different methods used for these results. are nearly isotropic. The forward hemisphere angular distributions of the relative cross sections for uranium at the same $\pi^{+}$ energy determined by track angles in SSNTD $^{28}$ have been normalized to the absolute uranium total cross sections of Hicks et $a l$. and added to this figure. Good consistency is found. Data and a fit for the lighter sample of Ho are also shown. ${ }^{28}$

4.3. Angular correlations between fission fragments. Fission fragments from binary fission at rest will be back-toback if no lighter particles are also ejected, but spread in angle depending upon the numbers and energies of such particles; the angular width is thus an indirect measure of the temperature of the fissioning system. ${ }^{1}$

The angular correlation between two fragments $\mathrm{W}(\theta)$ has been measured by several methods. The electronic methods of Hicks et al. ${ }^{48}$ gave the pattern shown in Figure 14 for $96 \mathrm{MeV}$ $\pi^{+}$, with widths (FWHM) of 21(1.5) deg. for U and 14(2) deg. for $\mathrm{Au}$. At $80 \mathrm{MeV}$, the SSNTD $\pi^{+}$data of Khan et al..$^{28}$ give the points shown for $\mathrm{Ho}, \mathrm{Au}$ and $\mathrm{Pb}$, compared to the $400 \mathrm{MeV}$ $\pi^{+}$data on uranium. ${ }^{53}$ These data have been adjusted to give the same area. The fits shown in Reference 28 yield FWHM for $80 \mathrm{MeV} \pi^{+}$on $\mathrm{Pb}$ and Ho to be $20.9 \mathrm{deg}$ and $14.4 \mathrm{deg}$, respectively. A measurement from a plot for the $400 \mathrm{MeV} \pi^{+}$ data on uranium ${ }^{53}$ gives a FWHM of $11.8 \mathrm{deg}$. It seems that this angular width is narrower for uranium at the higher pion

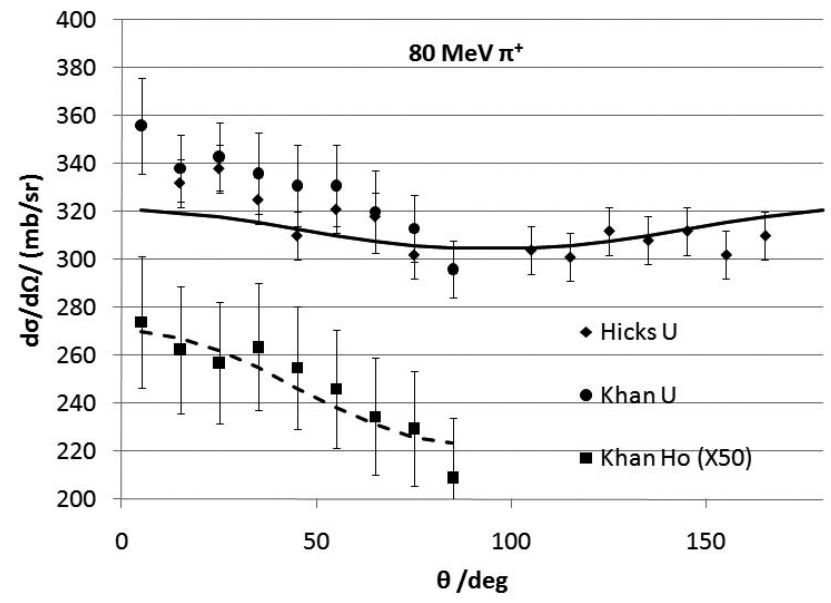

Figure 13. The angular dependence of fission cross sections induced in uranium and holmium by positive $80 \mathrm{MeV}$ pions was measured by Hicks et al. ${ }^{48}$ and Khan et al. ${ }^{28}$ using SSNTD, using different methods to obtain the angular information. Fits to the Hicks data using $A+B \cos ^{2}(\theta)$ are shown, with the parameters listed in Table 4. Holmium cross sections have been multiplied by 50 .

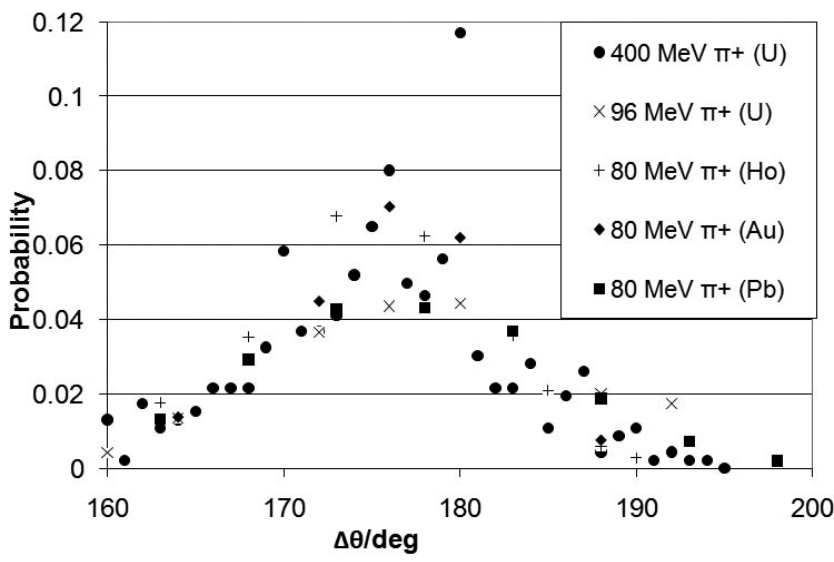

Figure 14. The angular spread between coincident fission fragments is plotted for $400 \mathrm{MeV} \pi^{+}$on uranium, ${ }^{53} 96 \mathrm{MeV} \pi^{+}$on uranium, ${ }^{48}$ and $80 \mathrm{MeV} \pi^{+}$on samples of $\mathrm{Ho}, \mathrm{Au}$ and $\mathrm{Pb}^{28}$ The width of these distributions is determined by the excitation energy of the compound system. 
beam energy. At $400 \mathrm{MeV}$ the pion mean free path in nuclei is longer than at $80 \mathrm{MeV}$, providing poorer coupling to excitation of the entire nucleus, and thus a lower temperature at scission for a narrower angular correlation.

Detailed calibration and examination of the tracks left in mica both upstream and downstream of gold and uranium samples by $400 \mathrm{MeV} \pi^{+}$beams were used to infer the forward momentum of the system at the moment of scission. ${ }^{53}$ These results are shown in Figure 15, with averages of $470 \mathrm{MeV} / \mathrm{c}$ for $\mathrm{U}$ and $214 \mathrm{MeV} / \mathrm{c}$ for $\mathrm{Au}$. The uranium nuclei held the pion beam momentum of $521 \mathrm{MeV} / \mathrm{c}$ more effectively than did the gold.

Fission events in uranium-loaded emulsion found tracks identified as protons in spatial coincidence with fission. ${ }^{46}$ Numbers of events for angles between two protons from beams of $280 \mathrm{MeV} \pi^{+}$and $460-660 \mathrm{MeV}$ protons in such coincidence are shown in Figure 16. Protons following proton-induced fission are isotropic, while those from pions show back-to-back or parallel trends. If the protons arose from $\pi^{+}$absorption on a quasideuteron, they would be back-to-back, while knockout protons from an energetic proton after absorption would be parallel. Figure 17 extends this study to measurements of the total energy of events with two or three protons in coincidence with fission. ${ }^{46}$ Back-to-back events from any one pair of protons carry more energy. This is solid evidence of the role of pion absorption leading to fission, with prompt protons after absorption carrying away much of the initial energy.

\section{Target Mass Dependences}

5.1. Overview. Considerations of the opposite effects of a Coulomb repulsion and an attractive liquid drop surface tension lead to the conventional use of the fissility parameter $Z^{2} / A$ as a plotting variable for fission from a state of charge $Z$ and mass $A$. It will be of interest in particular to examine the role of the pion beam charge in plots of $\sigma_{\mathrm{f}}(A)$, especially in the mass region from about gold to bismuth where fission cross sections at a fixed energy change rapidly with mass, with fission probabilities about one tenth of the reaction cross sections. How much of the beam charge is remembered at scission?

Data will be presented for three beam energy regimes - low energies where pion mean free paths are long, a $\Delta$ resonant energy near $150 \mathrm{MeV}$ with a short pion mean free path, and at $500 \mathrm{MeV}$ where mean free paths are again long and the higher energy could produce a wider range of reactions. Strings of data will be selected to show the mass dependence, in many cases using the same samples for all studies. All of the data shown will be from SSNTD, since this efficient method allowed up to ten samples to be exposed in the same beam simultaneously, decreasing the uncertainties due to beam normalizations. Then, each string of SSNTD was subjected to the

TABLE 5: Angular correlations

\begin{tabular}{ccclcc}
\hline Sample & Energy $(\mathrm{MeV})$ & $\theta_{0}(\mathrm{deg})$ & $\sigma(\mathrm{deg})$ & FLMT(deg) & Ref. \\
\hline${ }^{165} \mathrm{Ho}$ & 80 & 174.5 & 6.17 & 178.2 & 28 \\
${ }^{181} \mathrm{Ta}$ & 80 & 174.7 & 6.78 & 178.3 & 28 \\
${ }^{197} \mathrm{Au}$ & 96 & 176.0 & $5.96(0.85)$ & 178.4 & 48 \\
${ }^{\mathrm{nat}} \mathrm{Pb}$ & 80 & 175.6 & 8.64 & 178.7 & 28 \\
${ }^{238} \mathrm{U}$ & 96 & 178.5 & $8.94(0.63)$ & 178.5 & 48 \\
${ }^{238} \mathrm{U}$ & 400 & 175.0 & $5(1)$ & - & 53 \\
\hline
\end{tabular}

Angular correlation information for positive pion-induced fission is listed for several nuclei and several beam energies. The measured mean angle $\theta_{0}$ and the standard deviation $\sigma$ are given, and compared to the opening angle which would be provided if the full linear momentum of the beam had been retained to the point of scission (FLMT).

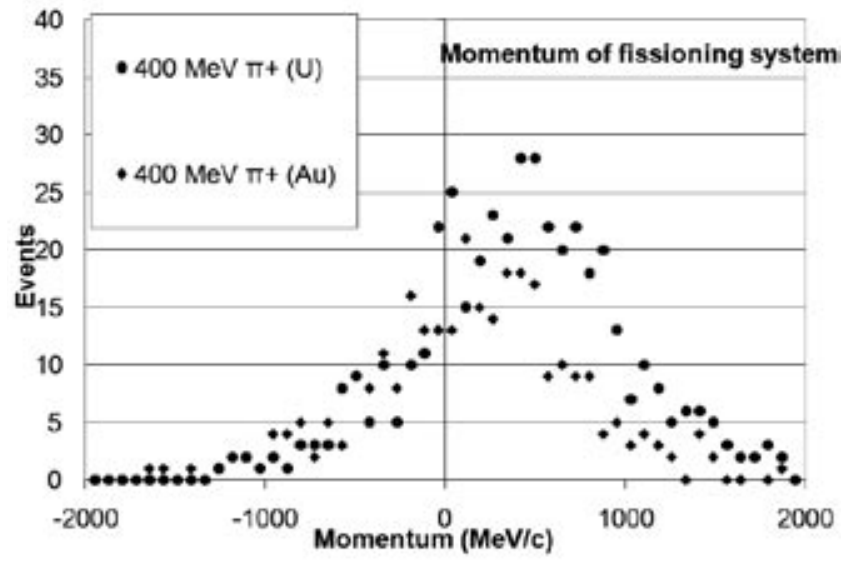

Figure 15. The coincident tracks left in mica SSNTD by fission fragments from $400 \mathrm{MeV} \pi^{+}$bombardment of uranium and gold have been analyzed to infer the forward momentum of the fissioning system at scission. ${ }^{53}$ The average values are $470 \mathrm{MeV} / \mathrm{c}$ for $\mathrm{U}$ and 214 $\mathrm{MeV} / \mathrm{c}$ for $\mathrm{Au}$.

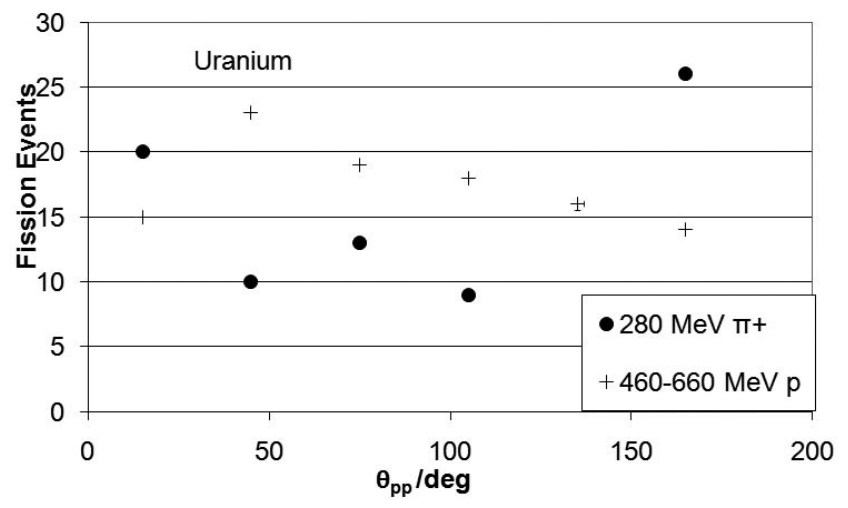

Figure 16. The distribution of events dependent upon the angle between pairs of proton tracks in emulsion in coincidence with fission is shown for events induced by $280 \mathrm{MeV} \pi^{+}$and by $460-660$ $\mathrm{MeV}$ protons. ${ }^{46}$ The coincident protons from proton-induced fission are isotropic, while those from pions are either back-to-back, as expected from pion absorption, or parallel, as might occur if one exiting proton knocked out another in the same direction. Events with both two and three proton tracks are included in this data set.

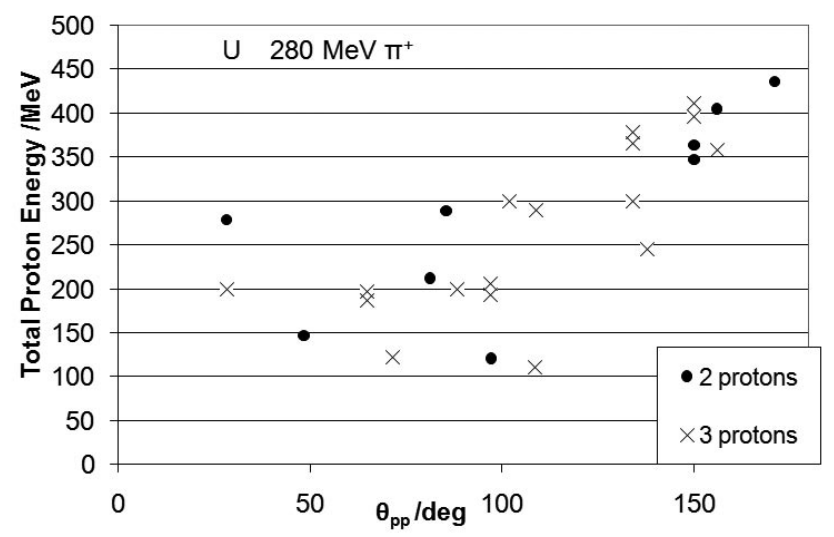

Figure 17. The energies of protons in emulsion observed in coincidence with $280 \mathrm{MeV} \pi^{+}$fission were summed in Reference 46 and are plotted here as a function of the opening angle between any two protons. Events with two and three protons are included in this data set. The highest energy events are those resulting from back-to-back events, as expected if pion absorption precedes fission to remove much of the available energy. The pion beam plus rest mass energy available is $420 \mathrm{MeV}$. 
same development and counting analysis, to diminish uncertainties due to these details. For instance, 'fission' events will have always been defined in the same way. All of the SSNTD samples used for these data were downstream from the samples. Measurements with bismuth samples were obtained from duplicated exposures at some energies, with good agreement.

In this review all pion cross sections $\sigma_{\mathrm{f}}(A)$ will be presented against the fissility parameter $(Z-1)^{2} /(A-2)$, with $Z$ and $A$ the charge and mass of the target system. If a positive pion is absorbed on a neutron, the resulting proton can be expected to depart promptly, accompanied by its proton partner from the quasideuteron pair in the opposite direction, as documented in Figure 16. The resulting charge is thus less by one. If a negative pion is absorbed on the proton member of the quasideuteron, two neutrons leave promptly, again for a net change of one charge loss. The less sensitive denominator in the fissility assumes the same process, with the initial loss of two nucleons from absorption. These expectations of charge and mass change are also suggested by the calculations of Iljinov. ${ }^{60}$

5.2. Cross sections. Figure 18 presents strings of data from simultaneous measurements of sample masses. Cross sections for negative pion beams at 131 and $138 \mathrm{MeV}$, as sensed in Makrofol SSNTD, are averaged for heavy nuclei, with positive beam data at $138 \mathrm{MeV}{ }^{23}$ The very thin $\left(20 \mu \mathrm{g} / \mathrm{cm}^{2}\right)$ actinide samples gave few counts, and this experiment had low statistical accuracy. Another exposure for both signs at $150 \mathrm{MeV}$ also used Makrofol, emphasizing the mass region where change is steepest. ${ }^{25}$ These data sets are at an energy where the delta resonance is expected to be dominant. Samples were about $1 \mathrm{mg}$ / $\mathrm{cm}^{2}$ thick, for good statistical accuracy.

Cross sections for positive pions at 80 and $100 \mathrm{MeV}$ are averaged for Figure 18, while a single datum for thorium is available for negative pions at $80 \mathrm{MeV}^{28,32}$ At these energies the mean free path is expected to be longer than that atop the resonance. Mica and CR39 SSNTD materials were used for these cross sections.

A negative pion beam at $500 \mathrm{MeV}$ provided the data points shown in Figure 18, again from a consistent exposure with many samples, including a few with isotopic enrichment ${ }^{205} \mathrm{Tl}$ and $\left.{ }^{206} \mathrm{~Pb}\right){ }^{24}$ These exposures also used Makrofol, and exactly the same target samples were used in common with the 131,138 and $150 \mathrm{MeV}$ exposures. ${ }^{23}$ Other more scattered measurements are not shown.

The general trend with mass (fissility) is seen to be a low and slowly changing cross section for the lighter nuclei, with a steep rise by a factor of one hundred from 28 to 32 , rising to nearly constant fission cross sections above $1000 \mathrm{mb}$ for the heaviest nuclei. For these heavy samples, $\pi^{-}$cross sections exceed those for $\pi^{+}$at the same energy.

Near the lead shell closure, with fissility near $32, \pi^{+}$cross sections exceed those for $\pi^{-}$at 150 and $138 \mathrm{MeV}$, a trend opposite to that for the heaviest samples. No direct comparisons are available at the same energies for the lighter nuclei.

Since the shell closure at ${ }^{208} \mathrm{~Pb}$ may influence these cross sections, Figure 19 shows an amplified view at $150 \mathrm{MeV}$, for both pion signs. A sequence of isotopically enriched samples was used for this experiment. ${ }^{25}$ Both beam signs show smooth upward trends of much the same average slope, interrupted in opposite senses by the case of ${ }^{208} \mathrm{~Pb}$ (with $(Z-1)^{2} /(A-2)=31.85$ ). Cross sections for $\pi^{+}$at 80 and $100 \mathrm{MeV}$ show the same upwards slope, but with the cross section for natural lead on that slope, defined by far fewer samples. ${ }^{28}$ This natural lead point, with four stable isotopes, lies just on the expected isotopic average for the $\pi^{+} 150 \mathrm{MeV}$ trend, reinforcing the dramatic difference noted for isotopic ${ }^{208} \mathrm{~Pb}$.

The beam sign dependence noted in Figure 19 indicates that $(Z-1)^{2} /(A-2)$ is not a fissility parameter that brings together data for the two beam signs. The trends are yet further apart using $Z^{2} / A$, and much better with $(Z+1)^{2} / A$ for $\pi^{+}$and $(Z-1)^{2} / A$ for $\pi^{-}$.

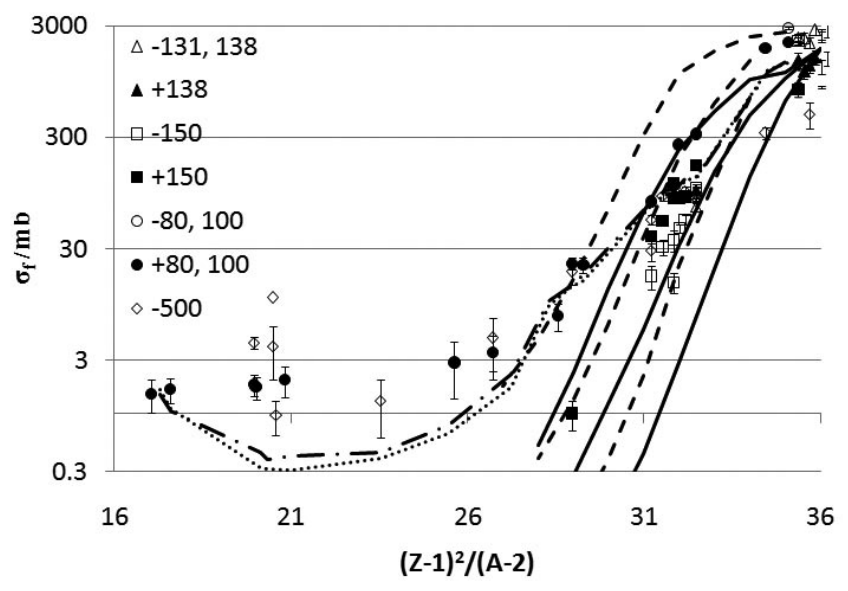

Figure 18. The mass dependences of reliably measured fission cross sections are shown as a function of the fissility parameter $(Z-1)^{2} /(A$ 2 ), assuming the loss of one unit of charge and two of mass after an initial two-nucleon absorption. Data are shown for $\pi^{+}$and $\pi^{-}$averaged for 131 and $138 \mathrm{MeV},{ }^{23}$ for $150 \mathrm{MeV} \pi^{+}$and $\pi^{-}$(Reference 25), for the average of 80 and $100 \mathrm{MeV} \pi^{+}$and $\pi^{-}$(Reference 28), and for $500 \mathrm{MeV} \pi^{-}$(Reference 26). The curves show the predictions for 80 $\mathrm{MeV}$ pions of both charges (solid for $\pi^{+}$, dashed for $\pi^{-}$) using ratios of level densities $a_{\mathrm{f}} / a_{\mathrm{n}}=1.1,1.05$ and 1.00 , from top to bottom. ${ }^{48}$ Many more sample masses at $80 \mathrm{MeV}$ are shown here than were available Reference 28. The dash-dotted (dotted) curves are for $500 \mathrm{MeV} \pi$, with similar parameters save for nuclear radii of $1.3(1.5) A^{1 / 3} \mathrm{fm}$ and the ratio of level densities $a_{f} / a_{n}=1.077(1.095) .{ }^{80}$

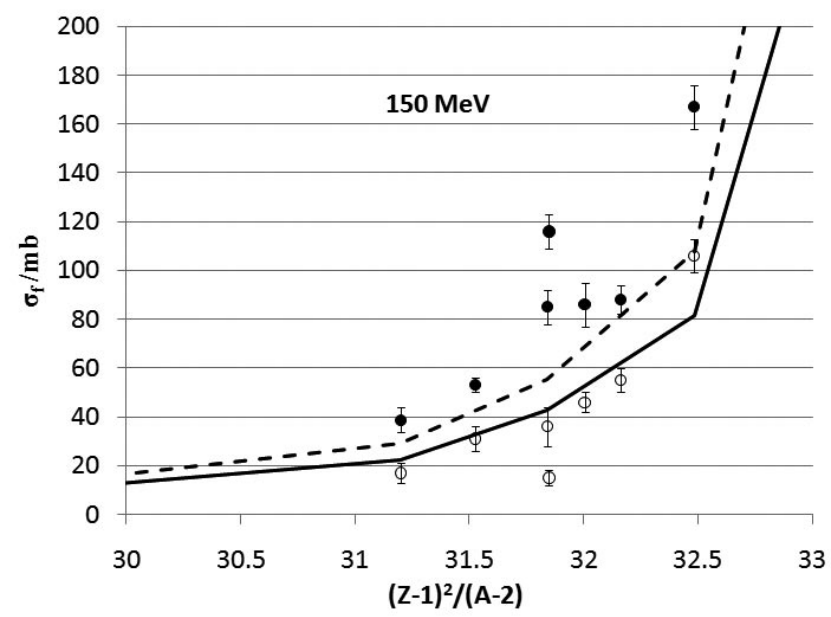

Figure 19. The use of isotopically separated targets for $\pi^{+}$and $\pi^{-}$ induced fission near doubly magic ${ }^{208} \mathrm{~Pb}$ at $150 \mathrm{MeV}$ permits a sharp discontinuity to be noted at that shell closure. ${ }^{25}$ A similar effect in found in the calculations for stopped $\pi^{-}$in Reference 65 . The solid $\left(\pi^{+}\right)$and dashed $\left(\pi^{-}\right)$curves show the fits to a wide range of results from Reference 26 .

This detailed dependence of cross sections upon beam sign and sample mass may serve as an important clue to the chain of processes from the initial collision to scission, and is largely consistent with the conclusion in Section 3 from the mass dependence of fragment TKE.

The expression fitted to a large range of nuclear fission probabilities from energetic beams in Reference 26 has been used with computed reaction cross sections $\sigma_{R}$ to compute the $\pi^{+}$ and $\pi^{-}$curves shown in Figure 19. The $\pi^{+}$data shown lie above their solid curve, while $\pi^{-}$data lie below their dashed curve.

The striking excursions seen in Figure 19 for ${ }^{208} \mathrm{~Pb}$ require examination, especially since exactly the same samples were used for both pion beam signs, and beam normalization and scanning techniques were the same within each sign. Perhaps $\sigma_{\mathrm{R}}$ dropped suddenly at ${ }^{208} \mathrm{~Pb}$, such that a fixed probability for fission leads to smaller cross sections. If so, other beam- 
induced fission cross sections should have noted this. More likely, the higher average nucleon binding for ${ }^{208} \mathrm{~Pb}$ deters some of the expected nucleon emission processes following the initial collision, permitting better, if partial, retention of the beam sign. In Section 7 a similar effect will be shown for fission induced by stopped $\pi^{-}$, and a theoretical comparison will be presented in Section 9.

However, an experiment measuring fission induced with quasi-monoenergetic neutrons on much the same range of nuclear isotopically separated samples found the fission cross section to rise smoothly with fissility, with no change from the trend for ${ }^{208} \mathrm{~Pb}^{61}$ If something unusual has happened to give the result shown here in Figure 19, it must be in the pion entrance channel.

Evaluated and averaged cross sections are presented for four nuclei with cross sections measured at several energies in Table 6. Some figures in the present work show data not listed in this table.

\section{Effects of Pion Beam Energy}

6.1. Introduction. Fission cross sections are available for at least some nuclei at beam energies from 65 to $2360 \mathrm{MeV}$, as listed in Table 6. Since pion-nucleon total cross sections show large resonant changes across this range, the mean free path of the pion in a heavy nucleus is expected to change, perhaps changing the coupling between the beam and nuclear excitations. At beam energies near $160 \mathrm{MeV}$, the prominent $\Delta$ resonance is found in free space and in light nuclei. Although observed reaction cross sections (Figure 1) show this feature to be damped in lead, we will seek a resonant effect in the fission channel. The role of pion absorption also changes with energy (Figure 2), and this could influence fission yields. At higher energies, single-nucleon knockout is common, but more energy is in principle available to the following reactions. At high energies the shorter Compton wavelength of an energetic pion should also have less overlap with the required pair of bound nucleons, leading to an expectation of less likely pion absorption. In this section the energy dependence of fission cross sections will be examined for a few nuclei with large data sets.
In many cases, the same nuclear target samples were used for each energy. Table 6 lists the sources of the data shown. Not all published data have been accepted for this Table.

6.2. Data. Figures 20 and 21 show fission cross sections for $\mathrm{Au}, \mathrm{Bi}$ and $\mathrm{U}$ (any isotope, including ${ }^{237} \mathrm{~Np}$ ) for positive pions, while Figures 22 and 23 shows results for negative pions. Very scattered data for tin are included. At the higher energies positive pion beams are contaminated by protons, and purely pion measurements are not possible.

Data for gold extend only up to $150 \mathrm{MeV}$ for $\pi^{+}$, but reach to $2360 \mathrm{MeV}$ for $\pi^{-66}$ Gold cross sections for $\pi^{+}$drop over the limited range available, while the $\pi^{-}$results rise by a factor of about three from lowest to highest energy. While the data show scatter, since they are derived from a range of techniques, the effect for $\pi^{-}$is striking. A similar trend is noted for $\pi^{-}$fission of tin, although counts are scarce and there is more scatter

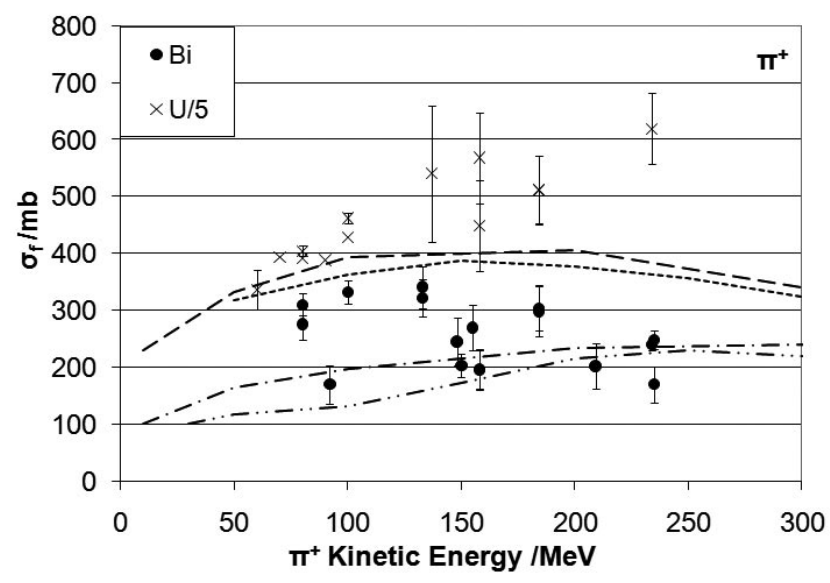

Figure 20. The positive pion beam energy dependence for fission is shown for several nuclei up to $300 \mathrm{MeV}$. Cross sections for uranium have been divided by five for simpler comparisons. Data are from several of the citations of this work, selected for best consistency. Theory curves for Bi and $\mathrm{U}$ are from CEM95 calculations of Mashnik ${ }^{27}$ (short dash and double-dot dash) and Yasin ${ }^{38}$ (long dash and dotdash).

TABLE 6: Cross section tables

\begin{tabular}{|c|c|c|c|c|c|c|c|c|c|c|c|c|c|c|}
\hline $\mathrm{T}(\mathrm{MeV})$ & neg $\mathrm{Sn}$ & uncert & pos $\mathrm{Au}$ & uncert & neg $\mathrm{Au}$ & uncert & pos Bi & uncert & neg Bi & uncert & pos $U$ & uncert & negU & uncert \\
\hline 60 & & & & & & & & & & & 1680 & 170 & 2960 & 370 \\
\hline 70 & & & & & & & & & & & 1970 & & 3000 & 300 \\
\hline 80 & 3.08 & 0.5 & 78 & 6 & & & 293 & 12 & 100 & 10 & 1990 & & 2830 & 150 \\
\hline 90 & & & 81 & 9 & & & & & & & 1940 & & 2260 & 230 \\
\hline 100 & 3.1 & 0.6 & 80 & 8 & & & 332 & 20 & & & 2225 & & 2360 & 240 \\
\hline 135 & & & & & & & 242 & 30 & 72 & 12 & 2700 & 600 & 2450 & 250 \\
\hline 150 & & & 47 & 6 & 21 & 4 & 224 & 25 & 122 & 10 & 2540 & 200 & 2740 & 230 \\
\hline 156 & & & & & & & 233 & 30 & & & & & & \\
\hline 184 & & & & & & & 298 & 44 & & & 2560 & & & \\
\hline 195 & & & & & & & & & 118 & 20 & & & & \\
\hline 212 & & & & & & & 202 & 40 & 73 & 20 & & & & \\
\hline 235 & & & & & & & 248 & 17 & 126 & 20 & & & & \\
\hline \multicolumn{15}{|l|}{280} \\
\hline 350 & & & & & 55 & 4 & & & 167 & 15 & & & 2300 & 300 \\
\hline 400 & & & & & & & & & 114 & 20 & & & & \\
\hline 500 & 7.5 & 3 & & & 47 & 6 & & & 148 & 50 & 1850 & 250 & & \\
\hline 672 & 37 & & & & 47 & & & & 89 & 11 & & & & \\
\hline 1068 & 23 & 6 & & & 135 & & & & 118 & 20 & & & & \\
\hline 1665 & 34 & 6 & & & 117 & & & & 136 & 20 & & & & \\
\hline 2360 & & & & & 107 & 20 & & & 191 & 40 & & & 1090 & 160 \\
\hline
\end{tabular}

Pion beam energy dependences of cross sections (in mb)for pion-induced fission are listed for a standard set of nuclei. In some cases published data have been averaged for this table. 


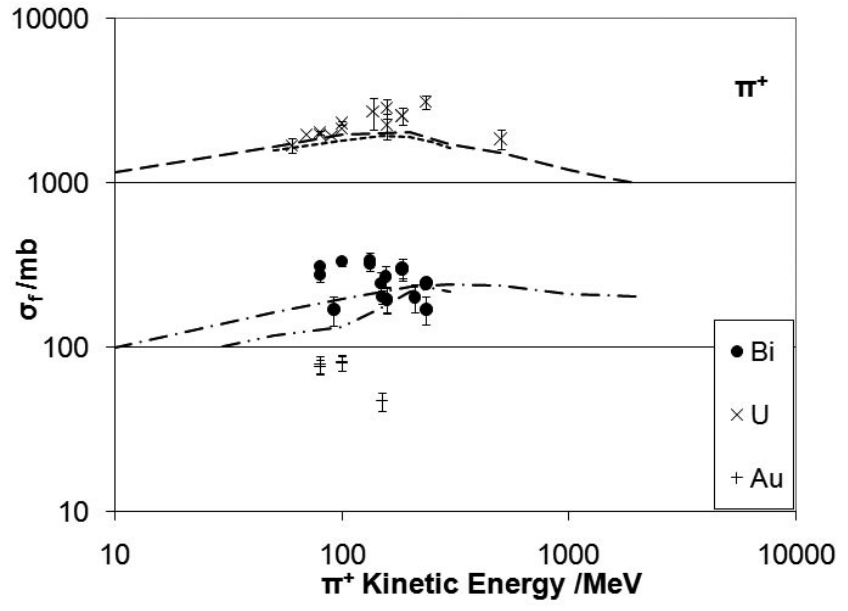

Figure 21. As Figure 20, but extended to the maximum pion energy considered for this work; negative pion data on the same scale are shown in Figure 23.

among the data.

Hicks shows the fission cross sections for gold from 60 to $100 \mathrm{MeV}$ for both pion signs. ${ }^{47}$ Negative pion cross sections at $60 \mathrm{MeV}$ are about twice those for $\pi^{+}$, but they become equal by $100 \mathrm{MeV}$. Reaction cross sections and those for pion absorption differ for the two charge states by factors of about 1.5 at $100 \mathrm{MeV}^{2}$ The fission cross sections for the two signs converge more rapidly than either reaction or absorption cross sections.

Positive pion fission cross sections for $\mathrm{Bi}$ are found to be remarkably constant from 80 to $234 \mathrm{MeV}$, except for one point at $90 \mathrm{MeV}$. Bismuth cross sections for $\pi^{-}$cover a much wider range of energies, but show much scatter, especially near 500 $\mathrm{MeV}$. These data will be compared to calculations in Section 9.

Positive pion fission cross sections for uranium rise with energy below $300 \mathrm{MeV}$, while those for $\pi^{-}$decrease. An adjustment by the Coulomb energy of $+/-16 \mathrm{MeV}$ is not enough to bring about agreement for the two charge states. For both charge states, the fission cross sections decrease at the higher energies, although data are sparse. The $870 \mathrm{MeV}$ data of Nishi indicate that spallation is an important reaction at higher pion energies. $^{43}$

These $\pi^{-}$data for $U$ and Bi are shown again in Figure 24, together with photofission cross sections on lead and uranium. ${ }^{62}$ Pion cross sections are larger by a factor of about 25 , and show less resonant structure than is noted in the photon data. A more useful comparison for these two beams is presented in the next section, using their respective probabilities for a reaction to yield fission.

\section{Fission Probabilities}

7.1. Introduction. In order to compare cross sections for energetic pions to measurements with stopped $\pi^{-}$, it is necessary to present data for energetic pions as fission probabilities $P_{f}=\sigma_{f} / \sigma_{R}$, the ratio to reaction cross sections. Here, these reaction cross sections are from calculations, as shown for lead in Figure 1. Use of these probabilities also allows comparisons to fission yields from stopped or energetic antiprotons, another particle which can be absorbed to deposit rest mass energy into the nuclear energy budget. An antiproton captured from rest in the typical heavy nucleus Mo creates an average of 4.85 pions, of which 2.21 will interact with the nucleus. ${ }^{63}$ Photofission cross sections are much smaller than those for strongly interacting beams, but with the help of fission probabilities, we can also compare data for this other absorbable field, which also will induce nuclear reactions by pion production. ${ }^{21}$

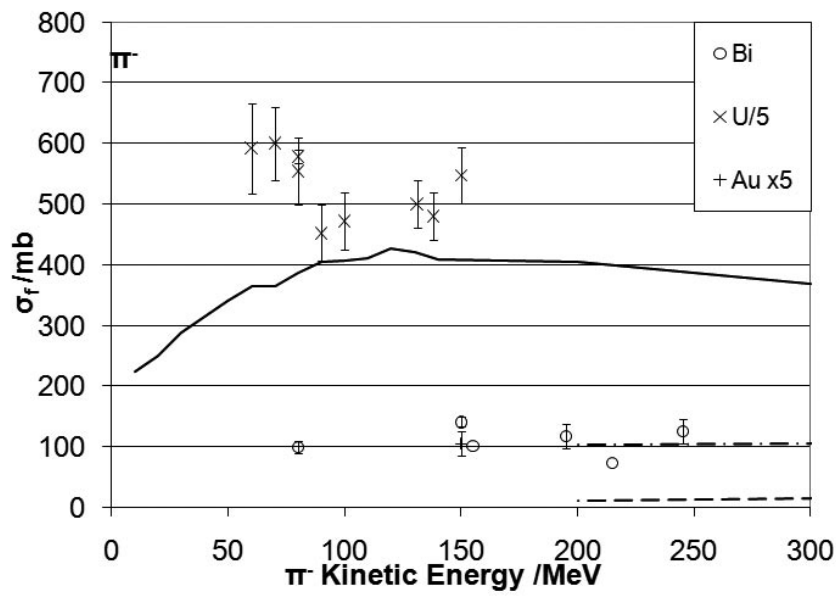

Figure 22. As Figure 20, but for negative pion beams up to $300 \mathrm{MeV}$. Curves are the CEM95 calculations from Yasin, solid for uranium, dot-dash for bismuth, and long-dashed for gold. ${ }^{35,36}$

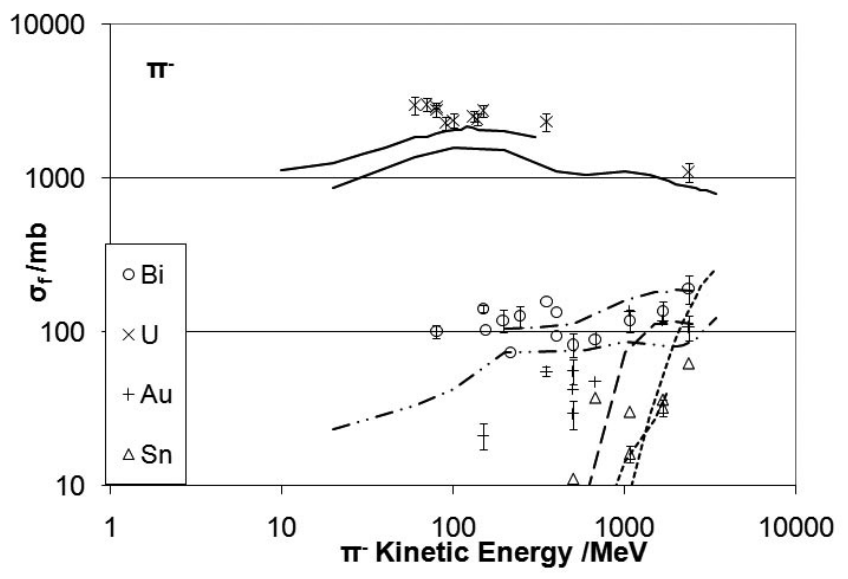

Figure 23. As Figure 22, extended to include the $2350 \mathrm{Mev} \pi{ }^{-}$data. ${ }^{66}$ Curves are as shown in Figure 22, plus the calculations of Mashnik, with the same code and different parameter usages, solid for uranium, short dash for tin, long dash for gold and dot dash and double dot dash for bismuth. ${ }^{81}$

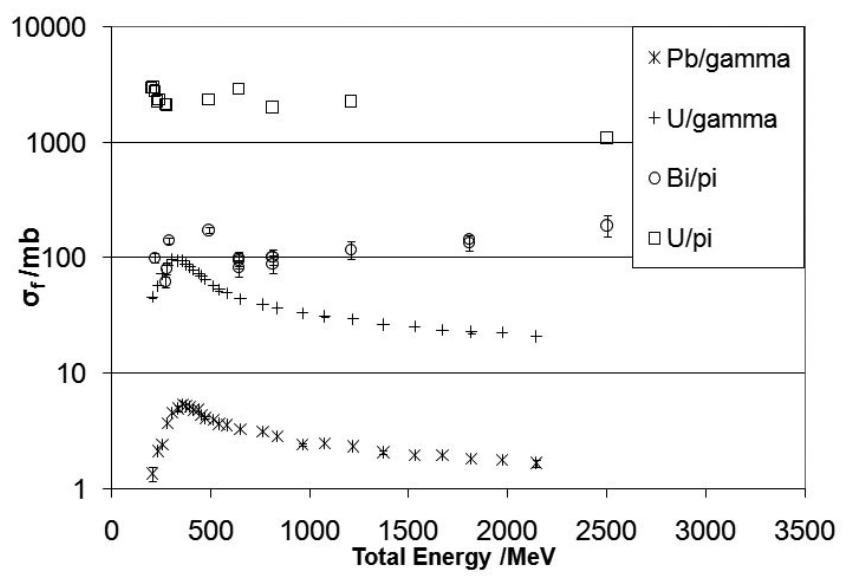

Figure 24. Fission cross sections are shown as a function of the total energy of the beam of photons ${ }^{62}$ or pions, with the pion scale including the $140 \mathrm{MeV}$ rest mass energy. The pion cross sections are larger by a factor of about 25 , and do not show the resonant structures found for photofission.

Pion reaction cross sections are computed in each case from DWIA calculations up to $300 \mathrm{MeV}$, using the code of Reference 8. At higher energies, $\sigma_{\mathrm{R}}$ is taken from the eikonal distorted wave code of Chen et al. in Reference 9. 
7.2. Energy dependences. Cross sections used here are the same as those presented in earlier sections. For better comparisons among beams, the energy scale is presented as the total energy of the beam, the kinetic energy as used in previous sections plus the rest mass energy of the pion $(140 \mathrm{MeV})$ or the antiproton and its annihilation partner $(2 \times 938 \mathrm{MeV})$. Figure 25 shows $\pi^{-}$data for bismuth and uranium, including the stopped pion data. ${ }^{51,64,65}$ The energy dependence for bismuth is smooth, extending from $220 \mathrm{MeV}$ to $2500 \mathrm{MeV}$. ${ }^{66}$ The curve will be described in Section 9. The uranium data are dense, with fission probabilities near unity, at or below $500 \mathrm{MeV}$, and a single datum at $2500 \mathrm{MeV}$ indicates a decrease in this fission probability. ${ }^{66}$

Photofission probabilities for gold, ${ }^{67}$ lead, and uranium ${ }^{62}$ are shown in Figure 26. Also shown are data for lead and uranium for stopped, ${ }^{19,58,68} 1.2 \mathrm{GeV}^{69}$ and $1.73 \mathrm{GeV}^{66}$ antiprotons. Reaction cross sections for energetic antiprotons are taken to be $\pi\left(1.4 A^{1 / 3}\right)^{2} \mathrm{fm}^{2}$. The photon and antiproton probabilities match well for $\mathrm{Bi}$ and $\mathrm{U}$, and compare closely with the pion fission probabilities in Figure 25, with all three beams showing constant probabilities for bismuth, and slowly decreasing trends for uranium.

7.3. Mass dependences. Figure 27 shows fission probabilities for $100 \mathrm{MeV}$ positve pions, ${ }^{28-31} 150 \mathrm{MeV}$ pions of both signs, ${ }^{25}$ and $500 \mathrm{MeV} \pi \cdot{ }^{-26}$ The $\mathrm{x}$-axis is taken to be $(Z-1)^{2} /(A-$ 2 ), as in Section 5. Also shown are the stopped $\pi^{-}$data from References 51 and 64. The solid curve is from a fit to proton induced fission probabilities for nuclei of the mass of tantalum and above, ${ }^{70}$ with the present usage of $(Z-1)^{2} /(A-2)$ :

$$
\mathrm{P}_{\mathrm{f}}=\exp [0.682\{(\mathrm{Z}-1) \ldots .36 .25\}] \text {. }
$$

The paper of Nix and Sassi also explores other theory-based equations for the mass dependence of proton-induced fission probabilities. $^{70}$

After nearly constant fission probabilities near $0.1 \%$ for energetic pions on lighter nuclei, the rapid rise to near unity at fissilities from 30 to 34 is matched by the curves shown, ${ }^{70,71}$ almost independent of the pion energies, but with $\pi^{+}$probabilities larger in the transition region. The dashed curve will be described in Section 9.

Fission probabilities for stopped, ${ }^{19,58,68} 1.2 \mathrm{GeV},{ }^{69}$ and 1.73 $\mathrm{GeV}^{66}$ antiprotons are shown in Figure 28 for a range of sample masses. The fissility axis is taken to be $(Z-1)^{2} /(A-1)$, assuming the annihilation of the antiproton on one proton. Also shown is a wide range of photofission data, using a range of SSNTD materials, ${ }^{49,72}$ with the fissility taken as $Z^{2} / A$. The energetic antiprotons are much more effective at inducing fission in lighter samples, but the trends of fission probabilities rise together near fissilities of 30 to 34. Energetic antiprotons are also more effective than stopped or energetic pions at inducing fission in lighter nuclei, by about a factor of ten. Energetic pions are more effective at inducing fission than photons, but photons are more likely to cause fission than stopped pions below a fissility of 28 .

The stopped $\pi^{-}$data of Reference 51 are shown again in Figure 29, and compared to theory curves as described in Section 9 below.

\section{Other Coincident Particles}

8.1. Introduction. In addition to observations of two traditional fission fragments, each near half the mass of the initial nucleus, measurements with pi mesons have sensed events with three fragments and events with several very light particles in spatial coincidence with fission. Only SSNTD and emulsion measurements have revealed these features, which are revealed from an understanding of the responses of the materials to particle charges and speeds.

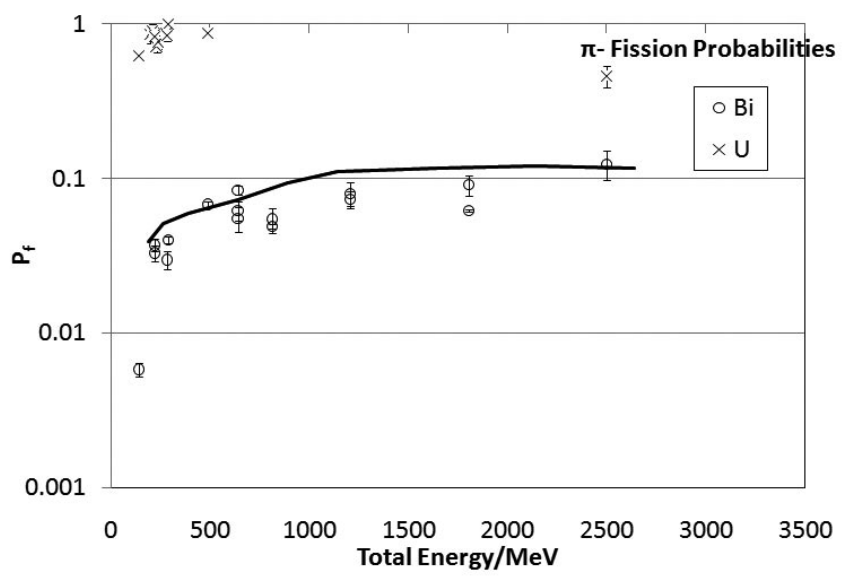

Figure 25. The probability for pion-induced fission as a fraction of the computed total reaction cross section $\sigma_{\mathrm{R}}$ is shown for bismuth and uranium. The solid curve is the Bi calculation of Yasin. ${ }^{36}$ The energy scale is the total energy of the beam.

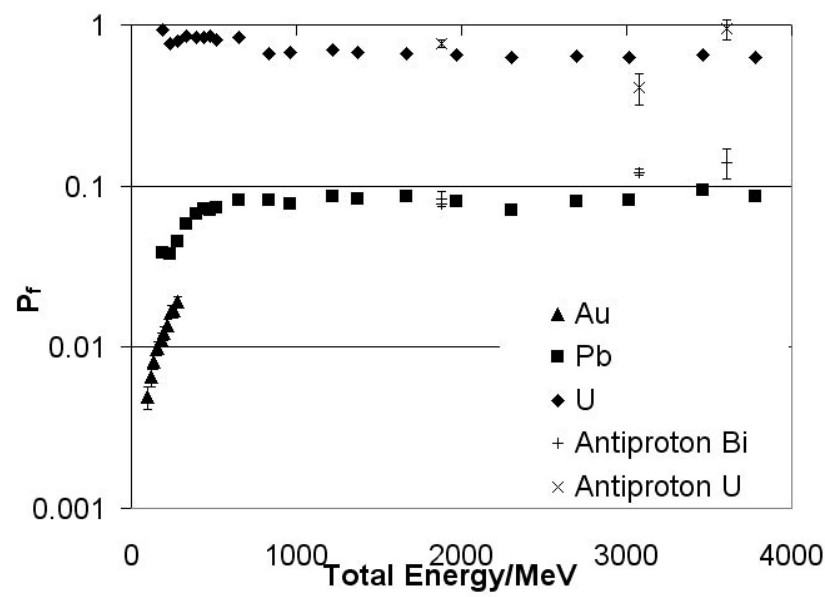

Figure 26. As Figure 25, but for photons on lead and uranium ${ }^{62}$ or gold. ${ }^{67}$ Also shown are results for stopped and energetic antiprotons on $\mathrm{Pb}$ and $\mathrm{U}^{19,66,68,69}$

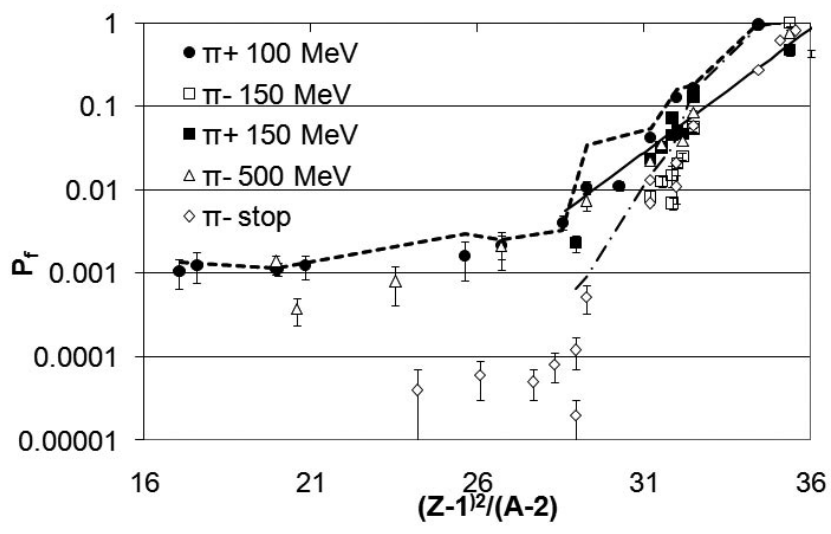

Figure 27. Fission probabilities are shown for $100 \mathrm{MeV}$ positive pions, ${ }^{28}$ pions of both signs at $150 \mathrm{MeV},{ }^{25}$ and $\pi^{-}$at $500 \mathrm{MeV}^{26}$ These are the data sets with the best consistency among those reported. Fission probabilities after the capture of a stopped $\pi^{-}$are also shown. ${ }^{51}$ The solid curve is from the equation in the text, as an early fit to proton-induced fission probabilities. ${ }^{70}$ The dot-dash curve is the CEM calculation of Reference 60 for $80 \mathrm{MeV} \pi^{+}$, translated to match the scale here of $(Z-1)^{2} /(A-2)$. The dashed curve shows the result of a CEM calculation for $100 \mathrm{MeV} \pi^{+}$, with $a_{\mathrm{f}} / a_{\mathrm{n}}=1.290$ for all nuclei. ${ }^{80}$

8.2. Ternary fission. The first paper reporting SSNTD observations of fission induced by pi mesons included observa- 


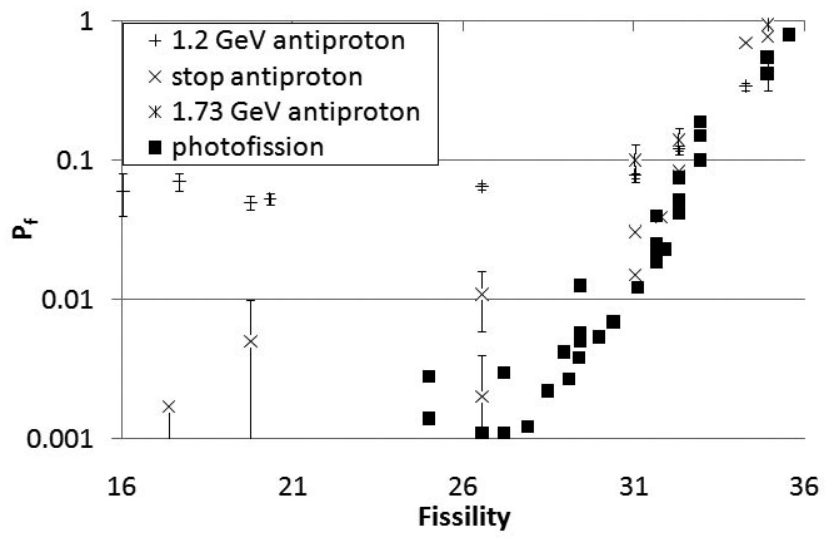

Figure 28. Photofission with a variety of techniques and over a wide range of bremmstrahlung photon energies was used to provide the fission probabilities shown ${ }^{72}$ while measurements with $1.2 \mathrm{GeV}^{69}$ and $1.73 \mathrm{GeV}^{66}$ antiprotons and three ${ }^{19,58,68}$ with stopped antiprotons yield the points shown for these beams. The charge for the $x$-axis is that appropriate to that of the initial absorption of the beam, the target charge $Z$ for photons and $Z-1$ for antiprotons.

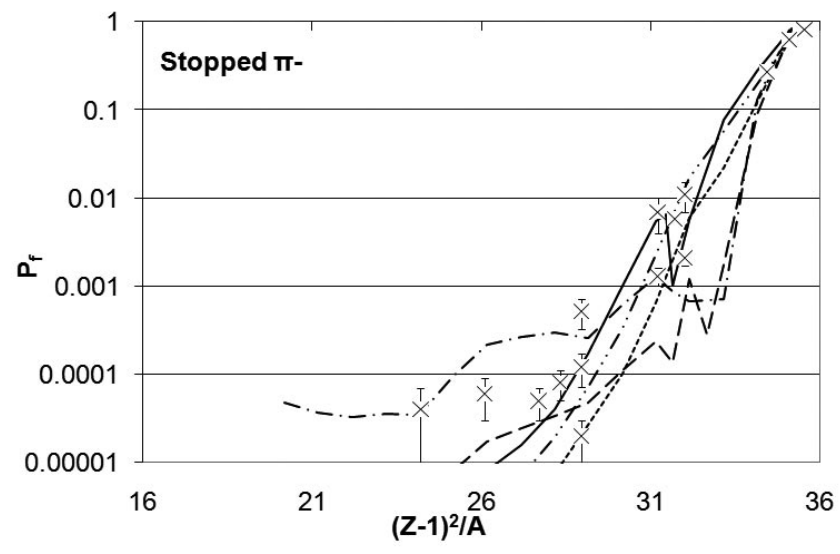

Figure 29. The probabilities for fission induced by stopped and captured $\pi^{-}$are shown ${ }^{51,64}$, and compared to five theoretical curves discussed in the text. The rapid changes near doubly-magic ${ }^{208} \mathrm{~Pb}$ are to be noted.

tions of ternary events, either by two correlated tracks in a $2 \pi$ system, or three tracks in a $4 \pi$ array of SSNTD. ${ }^{28}$ These and other results are presented in Figure 30, as the ratio of ternary to binary events $\sigma_{3} / \sigma_{2}$ for pions of energies from 80 to 2360 $\mathrm{MeV}^{28,29,66}$ Also shown in this figure is the same ratio, with the same methods as Reference 28 , for $7 \mathrm{GeV}$ proton beams. ${ }^{73}$ All cases show a decrease in this ratio for heavier nuclei, and the ratio is much the same for pions or protons, both pion signs, and all pion energies. Very similar ratios and the same trend were found with a beam of $1.73 \mathrm{GeV}$ antiprotons. ${ }^{66}$

The ratio of ternary to binary fission induced by heavy ion beams shown in Figure XIV-24 of the text of Vandenbosch and Huizenga ${ }^{1}$ shows just the opposite trend with sample mass, with $\sigma_{3} / \sigma_{2}$ rising to $3 \%$ for ${ }^{40} \mathrm{Ar}+{ }^{238} \mathrm{U}$. Although the heavy ion and pion reactions differ greatly in the angular momentum of the reaction systems, the magnitudes of the ratios are very similar. The conclusion from the heavy ion ratio is that cascade fission is the source of ternary events, in which one fission fragment in turn fissions. ${ }^{1}$ Since the overall mass distributions are similar for heavy ion and pion fission, they share a similar high excitation energy, making the similar results for $\sigma_{3} / \sigma_{2}$ reasonable.

8.3. Light reaction products. The early emulsion experiments showed that fission events were often accompanied by tracks for protons. Some of these protons show evidence of

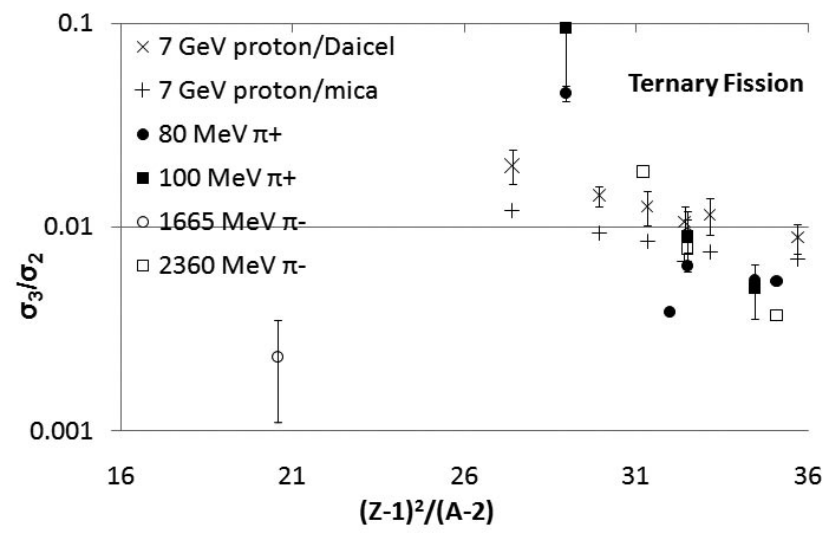

Figure 30. Ratios of ternary to binary fission yields are shown for a range of nuclei, for 80 and $100 \mathrm{MeV}$ positive pions ${ }^{28}$, for $1665 \mathrm{MeV} \pi^{-}$ ${ }^{33,34}$ and $2360 \mathrm{MeV} \pi \pi^{-66}$ Also shown are the same ratios for $7 \mathrm{GeV}$ protons, in two different SSNTD materials, Daicel and mica. ${ }^{73}$

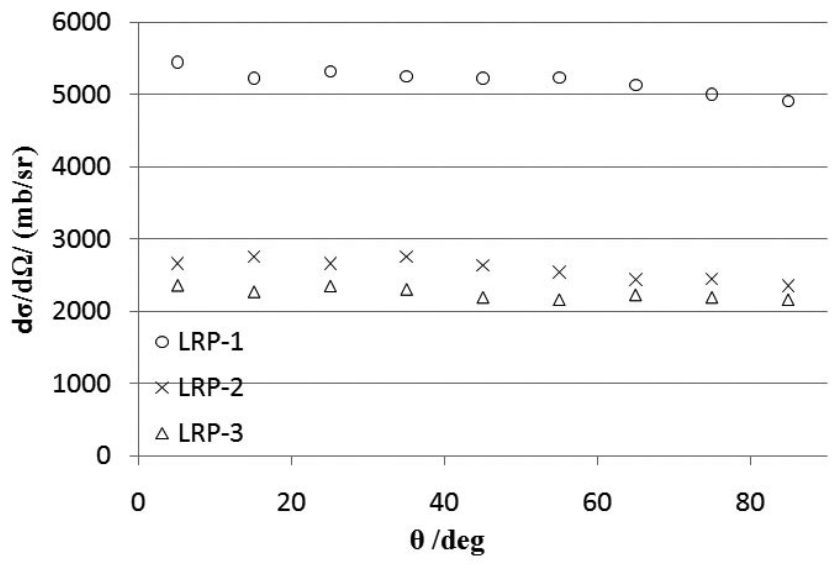

Figure 31. Light Reaction Product (LRP) tracks in SSNTD in spatial association with fission events from provide the angular distributions shown here. ${ }^{28}$ The relative cross sections of Reference 28 were normalized to the absolute fission cross sections of that work for this figure, for $80 \mathrm{MeV} \pi^{+}$on a uranium sample. LRP-1 events were from CR39, sensitive to charges of 1 and above, with proton energies below $1 \mathrm{MeV}$. LRP-2 were seen in CN-85, sensing $Z=2$ or greater, while LRP-3 events were measured in Lexan, sensing heavier fragments, with $Z$ between 8 and 16. Light charged particles in coincidence with fission are primarily protons. See Section 2 for a summary of the sensitivities of SSNTD materials.

arising from an initial absorption of the pion onto two or more nucleons. ${ }^{44,46}$ These results included very few events, examined in detail. For instance, a single long range $14 \mathrm{MeV}$ alpha particle was noted in pion fission of uranium, emerging nearly perpendicular to the axis between the fission fragments. ${ }^{44}$ This event is very similar to observations from many other studies with conventional beams. ${ }^{1}$ The fission tracks in emulsion were much the same for events with and without other charged particle tracks. ${ }^{44}$

Many more events of fission and associated Light Reaction Products (LRP) were presented by Khan et al. ${ }^{28,29}$ SSNTD materials were selected by their track thresholds in Reference 28 so as to measure yields of LRP, according to the sensitivities in Table 1. Since the $150 \mu$ mica SSNTD were between the nuclear sample and the plastic SSNTD, protons in the SSNTD were only registered above kinetic energies of $5 \mathrm{MeV}$, and alpha particles above $19 \mathrm{MeV}{ }^{29}$ Angular distributions for these fragments were determined in the forward hemisphere; these events were associated with fission tracks. Using the relative cross sections given in Reference 28 for fission, these LRP angular distributions have been normalized for Figure 31, showing an almost isotropic angular distribution. LRP-1 events 
from CR39 include protons, while LRP-2 (CN85) events must have charges at least of 2. LRP-3 events in Lexan include charges from 8 to 16 .

The angular distributions of these LRP are noted to be as isotropic as are the fission cross sections themselves. ${ }^{28,30}$ Two energetic protons emerging from absorption on a nucleon pair in bismuth have been shown to have a strongly anisotropic angular distribution, relative to the beam direction, with a forward or backward peak five times larger than that near $90^{\circ} .2$ The CR39 LRP-1 events include protons, with a cross section three times that for fission fragments, and show only a slight dip near $90^{\circ}$ for $80 \mathrm{MeV} \pi^{+}$on uranium. This SSNTD material would not register the high energy protons from simple twonucleon absorption. Evidently the pion reactions preceding fission involve more than two nucleons, with some low enough in energy to be registered in the SSNTD. The emulsion study for uranium with $280 \mathrm{MeV} \pi^{+}$did not report proton yields near 90 $\mathrm{deg}$, but did find the forward/backward ratio of protons registered in relativistic and P-9 emulsions to be 1.2(0.1). ${ }^{46}$ This is not inconsistent with an isotropic distribution for low proton energies as sensed in CR39.

Using SSNTD with different thresholds for registration of lightly ionizing particles, the data of Khan et al. ${ }^{28}$ may be used to generate a rough mass spectrum of the LRP. At least $80 \%$ of the LRP associated with fission events are protons for 80 $\mathrm{MeV} \pi^{+}$incident upon uranium, about $12 \%$ are alpha particles, and no more than $8 \%$ are heavier. Mass evidence from emulsion work is tainted by the many events from reactions on the $\mathrm{Ag}$ and $\mathrm{Br}$ in the emulsions, with tracks not associated with fission. ${ }^{46}$

The target mass $(A)$ dependence for $80 \mathrm{MeV} \pi^{+}$of the ratio of cross sections to produce LRP or fission fragments $\sigma_{\mathrm{LRP}} / \sigma_{\mathrm{f}}$ is shown in Figure 32, rising smoothly with $A{ }^{28,30}$ Over this range of $A$, the fission cross section has increased by a factor of more than one thousand, while pion absorption cross sections over this range of $A$ increase by about a factor of about three. ${ }^{2}$ Since the SSNTD used for these LRP determinations would not register the high energy protons arising from an initial twonucleon pion absorption, the LRP included in this figure probably arise during the chain of reactions between that initial step and fission.

\section{Theoretical Methods}

9.1. Introduction. Theoretical calculations for the observables from pion induced fission all follow methods as developed for explanations of more standard fission beams. Since the widths of pion-induced mass distributions indicate a high excitation energy at scission, a fairly simple approximation is valid and may be applied to the study of the fission barriers. Several versions of an IntraNuclear Cascade (INC) model have been developed and applied to pion-induced fission. These models require the adoption of several parameters, and the values of these parameters needed to match pion data may be compared to similar efforts with other beams. Another approach used a model for the establishment of a compound nucleus after the initial pion reaction, and then used a statistical model to compute the fission yields. Another presentation in this section will be of a general expectation of the role of multifragmentation to decrease fission yields, if such a process were to be established with pions. Finally, several methods have been used to compare different fission results with different beams, including pions.

9.2. The high energy approximation. The width of the mass distributions for fission fragments arising from pions in Section 3 indicates a high excitation at scission. ${ }^{1}$ We might therefore use a simple high energy approximation to estimate the probability for barrier penetration to yield fission. ${ }^{74,75}$ This was carried out for stopped, 80-100, and $500 \mathrm{MeV}$ pions in

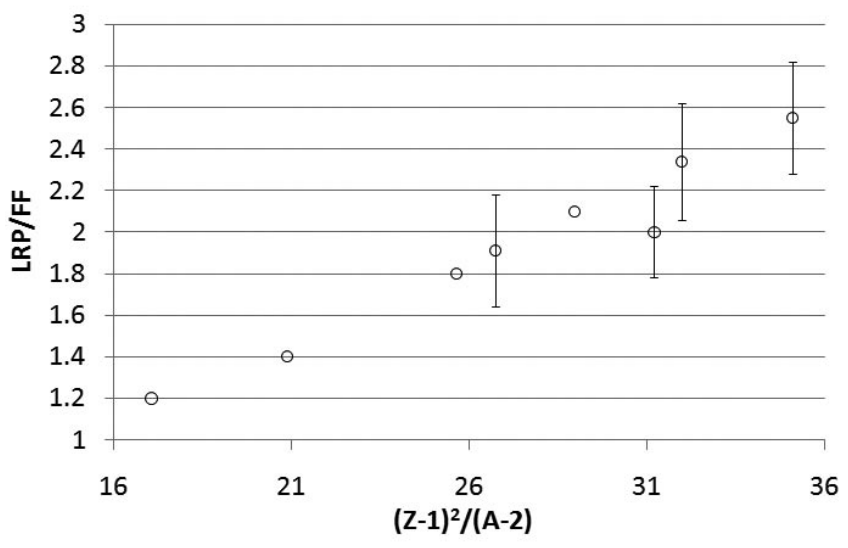

Figure 32. The ratio of cross sections for SSNTD observations of Light Reaction Products (LRP) to Fission Fragments (FF) is shown for a range of nuclei for $80 \mathrm{MeV}$ positve pions. ${ }^{28,30}$ Here, LRP are defined to have tracks from charge $Z<8$. Fission cross sections themselves increase by a factor of more than one thousand for this range of nuclei.

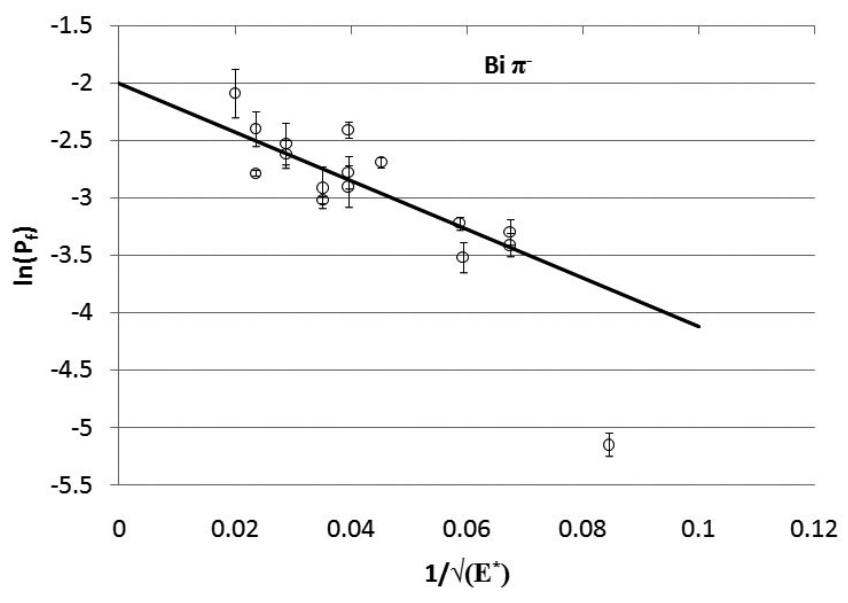

Figure 33. The high energy expression in the text suggests a plot of $\ln \mathrm{P}_{\mathrm{f}}$ for $\pi^{-}$on $\mathrm{Bi}$ vs. the reciprocal of the square root of the excitation energy at scission, as plotted here using the sum of pion kinetic and rest mass energies for $\mathrm{E}^{*}$. The line using the parameters in the text gives the slope with this same assumption, in good agreement with the data except for the datum for stopped $\pi^{-}$at the far right.

Reference 26, but is extended here.

The expression used is that the probability for fission is proportional to an exponential with the inverse of the square root of the excitation energy of the initial system, $E^{74,75}$

$$
P_{\mathrm{f}} \sim \mathrm{a} / A^{2 / 3} \exp \left[-\left(\mathrm{B}_{\mathrm{f}}-\mathrm{B}_{\mathrm{n}}\right) \sqrt{ }(a / E)\right] .
$$

Only data for bismuth will be shown here, with the parameters as used in Reference 26: level density $a=19 \mathrm{MeV}^{-1}$, fission barrier $B_{\mathrm{f}}=8.8 \mathrm{MeV}$ and neutron binding energy $B_{\mathrm{n}}=3.94 \mathrm{MeV}$. Data for $\pi^{-}$are shown in Figure 33, using the sum of pion kinetic and rest mass energies to compute $E$. The data for $P_{\mathrm{f}}$ are those of Figure 25, and include the stopped pion datum at $E=140 \mathrm{MeV}$. Except for that point, furthest to the right on the scale, the $\pi$ fission probabilities of Bi do fall along the computed line, starting at $P_{\mathrm{f}}=13.5 \%$ at an infinite energy, with the slope given by the high energy approximation expression.

This agreement is surprising, since evidence has been presented in previous sections that energetic protons carry off much of the initial total energy. If, however, the energy retained to fission is the same fraction of the initial total energy, the data and the line in Figure 33 would shift together to the right under this assumption. The datum for the stopped $\pi^{-}$remains as an anomaly. 
The high energy approximation was also used in Reference 26 for the mass dependence of $P_{\mathrm{f}}$. For nuclei lighter than bismuth, the mass dependence of $P_{\mathrm{f}}$ was reproduced using the full energy, kinetic plus rest mass, of the pions for $E$.

9.3. The compound nucleus and statistical fission. One way to compute probabilities for pion-induced fission is to consider a two-step process, in which the initial pion reaction forms a compound nucleus which later decays. This is the approach of Reference 76, in which a stopped $\pi^{-}$is assumed to be absorbed on a pair of nucleons. One of these nucleons leaves, while the other reacts to form the compound system. The computed spectrum of fast neutrons from $\pi^{-}$stopped on gold was found to agree with measurements. The fission decay of the compound nucleus was found to be steeply dependent upon the ratio of level densities of states leading to fission $a_{\mathrm{f}}$ to that for neutron decay $a_{\mathrm{n}}$. Data available at the time demanded that $a_{\mathrm{f}} / a_{\mathrm{n}}$ be in the range of 1.1 to $1.4 .^{76}$

Hicks used two means to establish a compound nucleus as a first step to fission. ${ }^{47,48}$ In one, an optical model including twonucleon absorption of the pions was used to generate a total absorption cross section; this method used fits to elastic pion scattering at similar angles to establish some parameters, as described in Reference 48. The second means was to use the computer code ISOBAR, ${ }^{77}$ which assumed the formation of a delta resonance. In either case, the decay of the compound nucleus to fission was computed with the code ALICE. ${ }^{78}$ As noted in Reference 76, the results are very sensitive to the ratio $a_{\mathrm{f}} / a_{\mathrm{n}}$. Over the limited range of beam energies in Reference 48 , use of the optical model method and $a_{\mathrm{f}} / a_{\mathrm{n}}=1.1$ was able to reproduce the magnitude and energy dependence of fission of uranium with both beam signs. Too few nuclear targets were used to test these models over a significant range of $A$. Curves show these predictions compared to data in Figure 18 for several ratios $a_{\mathrm{f}} / a_{\mathrm{n}}$.

9.4. IntraNuclear Cascade (INC) calculations. The most recent calculations for pion-induced fission cross sections have used the Cascade -Exciton Model code CEM. ${ }^{79}$ In this model, no pion absorption occurs, only a sequence of scattering reactions. The model assumes three stages - a first reaction to create an excited nucleus, including emission of fast or preequilibrium nucleons, then a compound nucleus is allowed to emit slow nucleons, and the chain ends with fission. Almost all of the parameters of these calculations were set by forcing agreement with proton and fast neutron-induced fission, as in Reference 79. This model has been applied to an analysis of all features of captured $\pi$, save for the fission channel. ${ }^{3}$ The one parameter which was varied in the comparisons to data in Reference 27 was the ratio of the surface area of the fissioning nucleus to the surface area of an equivalent sphere, $B_{\mathrm{s}}$. Proton(neutron)-induced fission of bismuth required $B_{\mathrm{s}}=1.18(1.12)$, but values of 1.12 and 1.15 were used for bismuth and uranium, respectively, to yield the curves compared to the beam energy dependence of the $\pi^{+}$-induced fission of these nuclei in Reference 27. The bismuth data were matched, but the uranium data above $100 \mathrm{MeV}$ were above the calculated values.

Yasin applied the same CEM model and code to $\pi^{-}$fission of $\mathrm{Au}$ and $\mathrm{Bi}$ over a wider range of beam energies. An energydependent ratio of level densities $a_{\mathrm{f}} / a_{\mathrm{n}}$ was derived from previous fission studies. ${ }^{35}$ Parameter choices were confirmed by computing the proton beam energy dependence of $\mathrm{Au}, \mathrm{Pb}$ and $\mathrm{Bi}$ fission. Excellent agreement was found for the magnitudes and energy dependence of $\pi^{-}$-induced fission of $\mathrm{Au}$ and $\mathrm{Bi}$. This effort was extended to the light systems Sn and Ho in Reference 34, again with good success, to uranium in Reference 37, and to actinides in Reference 38. Fission induced by $\pi^{+}$was compared to CEM calculations in Reference 38, again with good success for a limited range of beam energies. A summary of these pion results and a comparison to data and calculations

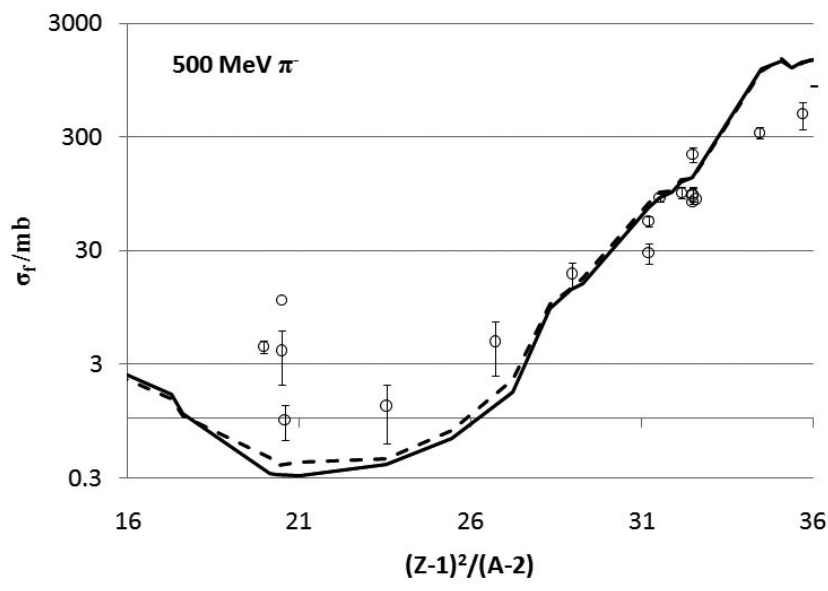

Figure 34. The cascade exciton model (CEM) of Reference 79 has been used to compute fission induced by $500 \mathrm{MeV} \pi^{-}$across a wide range of nuclei. Here the results are compared to a compilation of data at this beam energy. The two curves differ only in the ratio of level densities $a_{\mathrm{f}} / a_{\mathrm{n}}$, with 1.095 for the solid curve and 1.077 for the dashed curve. Parameters were adjusted so as to match a cross section of $132 \mathrm{mb}$ for ${ }^{209} \mathrm{Bi}$.

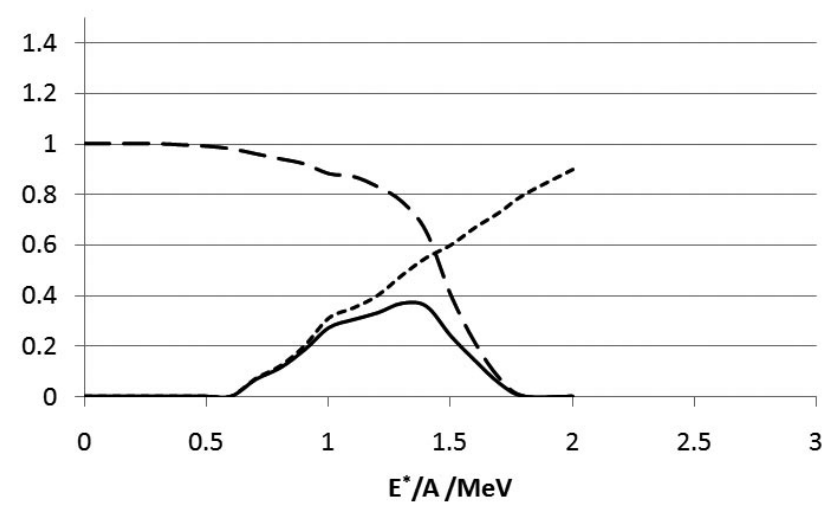

Figure 35. The long-dashed curve shows the sum of probabilities that a multifragmentation reaction on bismuth would leave a heaviest nucleus of mass at least $201 .^{81}$ The short-dash line shows the fission probability for such nuclei (times 10), averaged over nuclei with masses between 201 and 209, and averaged for several pion beam energies. The solid line is proportional to the product of these curves, or the probability of fission occurring after a multifragmentation process. The horizontal axis uses an excitation energy $E^{*}$ equal to the pion mass plus beam energy. The sharp drop near $E^{*} / A=1.4 \mathrm{MeV}$ suggests that multifragmentation would cause a sharp change in fission probabilities for bismuth at beam energies near $150 \mathrm{MeV}$. No such effect is noted in Figure 25, with data from an experiment carried out to examine just this idea.

from the CEM code for pions, photons, protons and neutrons is found in Reference 39. A recent calculation used a fixed ratio $a_{\mathrm{f}} / a_{\mathrm{n}}=1.290$ in a CEM calculation of the fission probabilities for $100 \mathrm{MeV} \pi^{+}$on a wide range of nuclei. ${ }^{80}$ Results are compared to the data in Figure 27, finding a good match to the trend of a wide range of nuclei.

The CEM model might be expected to be more reliable at higher beam energies, with a longer chain of collisions within a heavy nucleus. Figure 34 shows the fission data (as before) for $500 \mathrm{MeV} \pi$ for a range of nuclei, compared to CEM calculations with two ratios of level density parameters. ${ }^{35-39,81}$ The computed cross sections were tied to a bismuth cross section of $132 \mathrm{mb}$ to set them to the same scale. The mass dependences of the calculations are very similar, and match the general trend of the data.

In contrast to the CEM calculations that did not include the absorption mechanism, fission induced by pions absorbed after capture from atomic orbits has only this means to couple the 
rest mass energy into the nucleus. Iljinov et al. used a model in which this is the only beginning of the reaction chain leading to fission. ${ }^{71}$ This work computed the spectra and multiplicities of protons and neutrons, the distributions of final nuclei (largely by spallation), and fission probabilities from $\pi^{-}$capture from atomic orbits. A model based on absorption onto an alpha particle, with decay using $a_{\mathrm{n}} / a_{\mathrm{f}}=1.00$, gave adequate agreement with the mass dependence of fission probabilities. A CEM model was also used to compute fission probabilities induced by stopped negative pions for a wide range of nuclei in Reference 65 . A discontinuity was noted near doubly-magic ${ }^{208} \mathrm{~Pb}$, and ascribed to the rapidly changing fission barriers. A similar effect for $150 \mathrm{MeV}$ pions is shown in Figure 19 and was discussed above.

With a matched set of excitation energies from beams of photons, nucleons and pions, fission probabilities of $\mathrm{Au}, \mathrm{Bi}$ and $\mathrm{U}$ were computed and compared in Reference 60 . These probabilities were much the same for these three means of exciting the nucleus, and in agreement with the expectations from a CEM code.

It appears that the modern CEM codes can account for the mass and energy dependences of pion induced fission, with only a few small changes in parameters from more traditional fission studies. Other features of reactions induced by the pions are also accounted for, all without any need for unusual mechanisms. It is interesting to note from the calculations that pion-induced fission yields are very tolerant of the assumed initial pion-nucleus interaction.

9.5. Multifragmentation. One of the initial goals of pioninduced fission was to seek evidence of very high excitation energies all the way to the point of scission, indicating the retention of the pion rest mass energy all the way through the chain of reactions. Another reaction mechanism at high excitations would be in competition with fission, but also produce heavily ionizing particles. This is multifragmentation or 'cracking', in which an energetic heavy nucleus disassembles into fragments of individually tight binding energy. ${ }^{82}$ Lacking the strong Coulomb repulsion of fission, these fragments would not be registered as fission fragments. However, this fragmentation would be a strong source of loss of nuclear charge $Z$, greatly decreasing the probability of fission itself. Since multifragmentation is expected to be a phase change, a rapid change of observables with energy might be expected. Detailed studies of the beam energy dependence of fission cross sections might reveal such a change. An example at one energy is the mass distribution noted for fragments from $870 \mathrm{MeV} \pi$ on $\mathrm{Bi}^{43}$

The multifragmentation code of Bondorf ${ }^{82}$ was run by colleagues in Rio de Janeiro ${ }^{83}$ for bismuth. Figure 35 shows as a dashed line the sum of probabilities that the heaviest fragment has a mass of at least 201, as a function of the nuclear excitation per nucleon $E^{*} / A$. We assume lighter nuclei will have very low fission probabilities, as judged from the rapid mass effect shown in Figure 18. The dotted line shows a smooth curve of the fission probabilities for bismuth with $\pi^{-}$, using as the excitation energy per nucleon $E^{*} / A$ the sum of pion kinetic and rest mass energies. The product of these curves would be the probability that multifragmentation leaves enough mass to have a reasonable probability to fission. A curve proportional to this product is shown as the solid curve, with a sharp energy dependence near a pion kinetic energy of $150 \mathrm{MeV}$. Although this curve is the result of several strong assumptions, the dramatic change suggests that a multifragmentation process might be observable in the fission channel.

A detailed excitation function for $\pi^{+}$fission of bismuth was carried out to seek such a change, ${ }^{27}$ but no changes from a smooth trend were noted for beam energies from 92 to 234 $\mathrm{MeV}$. This indicates that even the predicted fast multifragmentation process ${ }^{82}$ is not so rapid as to occur before much of the pion initial energy was lost by other means, such as absorption and emission of energetic nucleons before an overall nuclear excitation could be formed.

\section{Conclusions}

A wealth of scattered information on the observables of nuclear fission following pions of both signs incident upon nuclei has been collected over many years. Fission cross sections (or probabilities) and the angular distributions and correlations of fragments have been measured by several techniques. Overall, the observations agree, and calculated values using modern theories and codes agree with the data. We may thus accept outputs of these codes, using parameters as tested with data as shown in this work, to generate outputs for future needs. Light particles in association or coincidence with fission have been observed, showing evidence of the initial absorption of the pion into the nucleus, as well as evidence of lower energy products on the path from the absorption to fission. These prompt processes remove so much of the initial energy from the rest mass and kinetic energies of the pions that the final fission shows features much as observed with other energetic beams, including photons and antiprotons, with very similar trends in their fission probabilities. Several very different reaction models give predictions that match the fission cross section data, indicating that this observable is very tolerant of the assumed reaction mechanism.

The data shown in this work are consistent enough and complete enough, and in sufficient agreement with theory, that further experimental work seems not to be required. This review probably closes a chapter, uniting the exotic pion-nucleus reaction possibilities with the familiar processes and observables of nuclear fission.

Acknowledgements. It is a pleasure to thank the friends and colleagues who have worked with me on this decades-long project. The team in Rio de Janeiro was led by Profa. Solange de Barros, and that in Pakistan was ably directed by Dr. Hameed Ahmed Khan. The more recent energetic efforts of Dr. Zafar Yasin are deeply appreciated. Vital help and wisdom in the theory efforts were provided by Dr. Stepan Mashnik, and Jonathon Morrow gave much help with the figures. This work was supported in part by the USDOE and the US National Science Foundation.

\section{References}

(1) R. Vandenbosh and J. R. Huizenga, Nuclear Fission (Academic Press, New York and London, (1973).

(2) D. Ashery and J. P. Schiffer, Ann. Rev. Nucl. Part. Sci. 36, 207 (1986).

(3) S. G. Mashnik, Rev. Roum. Phys., Tome 37, 179 (1992).

(4) B. W. Allardyce et al., Nucl. Phys. A 209, 1 (1973).

(5) D. Ashery et al., Phys. Rev. C 23, 2173 (1981).

(6) C. J. Gelderloos et al., Phys. Rev. C 62, 024612 (2000).

(7) R. J. Peterson, Few-body Systems Supp. 9, 17 (1995).

(8) A. A. Ebrahim and R. J. Peterson, Phys. Rev. C 54, 2499 (1996).

(9) C. M. Chen, D. J. Ernst, and M. B. Johnson, Phys. Rev. C 48, 841 (1993).

(10) SAID--- // gwdac.phys.gwu.edu

(11) M. K. Jones et al., Phys. Rev. C 48, 2800 (1993).

(12) K. Nakai et al., Phys. Lett. 121B, 373 (1983).

(13) K. Nakai, Nucl. Phys. A 418, 163c (1984).

(14) W. C. Hsi et al., Phys. Rev. Lett. 79, 817 (1997).

(15) R. D. McKeown et al., Phys. Rev. Lett. 44, 1033 (1980).

(16) G. E. Brown et al., Phys. Let. 118, B 39 (1982).

(17) M. B. Golubeva et al., Phys. Lett. B 221, 238 (1989).

(18) J. N. Knudson et al., Phys. Rev. C 44, 2869 (1991).

(19) T. Von Egidy et al., Nucl. Phys. A 558, 383c (1993).

(20) M. Kh. Khankhasayev, in Nuclear Methods for Transmutation of Nuclear Waste (World Scientific, 
Singapore, ed. M. Kh. Khankhasayev, H. S. Plendl and Zh. B. Kurmanov, 1996).

(21) J. D. T. Arruda-Netto et al., Phys. Rev. C 54, 3294 (1996).

(22) J. H. Kim and H. Toki, Prog. Theor. Phys. 72, 1050 (1984).

(23) S. de Barros, I. O. de Souza, M. B. Gaspar, and R. J. Peterson, Nucl. Phys. A 542, 511 (1992).

(24) S. de Barros et al., Proc. Brazilian Physical Society, Angra dos Reis (1996).

(25) S. de Barros, J. C. Suita, A. G. Da Silva, and R. J. Peterson, Z. Phys. A 359, 35 (1997).

(26) R. J. Peterson, S. de Barros, I. O. de Souza, M. B. Gaspar, H. A. Khan, and S. Manzoor, Z. Phys. A 352, 181 (1995).

(27) R. J. Peterson et al., Eur. Phys. Journal 10, 69 (2001).

(28) H. A. Khan, N. A. Khan, and R. J. Peterson, Phys. Rev. C 35, 645 (1985).

(29) H. A. Khan and R. J. Peterson, Presented at $14^{\text {th }}$ Int. Conf. on SSNTD, Lahore, Pakistan (1988).

(30) H. A. Khan and R. J. Peterson, Nucl. Instrum. Methods Phys. Res. B 51, 421 (1990).

(31) H. A. Khan, N. A. Khan, and R. J. Peterson, Phys. Rev. C 43, 250 (1991).

(32) H. A. Khan, N. A. Khan, and R. J. Peterson, Int. J. Radiat. Appl. Instrum. D 19, 637 (1991).

(33) H. A. Khan et al., Radiat. Meas. 28, 287 (1997).

(34) H. A. Khan et al., Radiat. Meas. 43, S188 (2008).

(35)Z. Yasin, M. I. Shahzad, I. E. Qureshi, G. Sher, and R. J. Peterson, Nucl. Phys. A 765, 390 (2006).

(36)Z. Yasin, M. I. Shahzad, R. J. Peterson, G. Sher, M. T. Javed, and H. A. Khan, Nucl. Phys. A 781, 296 (2007).

(37)Z. Yasin, R. J. Peterson, M. I. Shahzad, and H. A. Khan, Proc. Int. Conf. on Nuclear Data for Science and Technology, 1137, (EDP Sciences, ed. O. Bersillon, F. Gunsing, E. Bauge, R. Jacquin, and S. Leray, 2007).

(38) Z. Yasin, M. I. Shahzad, H. A. Khan, R. J. Peterson, and M. Asghar, Radiat. Meas. 43, 174 (2008).

(39)Z. Yasin et al., Radiat. Meas. 44, 846 (2009).

(40) E. K. Hyde, Nuclear Properties of the Heavy Elements, Vol. III, Fission Phenomena, (Prentice-Hall, Englewood Cliffs, NJ, 1964).

(41) B. J. Dropesky, Los Alamos Report LA-8151-PR (1979).

(42) R. J. Peterson, Nucl. Phys. A 769, 95; ibid. 115 (2006).

(43) T. Nishi et al., Bull. Inst. Chem. Res. Kyoto Univ. 60, 132 (1982).

(44) G. E. Belovitskii et al., Soviet Physics JETP 2, 493 (1956).

(45) G. F. Denisenko et al., Phys. Rev. 109, 1779 (1958).

(46) J. S. Ivanova, Sov. Phys. JETP 34, 955 (1958).

(47) K. H. Hicks, Ph. D. thesis, University of Colorado (1984).

(48) K. H. Hicks et al., Phys. Rev. C 31, 1323 (1985).

(49) D. A. De Lima, J. B. Martins, and O. A. P. Tavares, Nucl. Instr. Methods B 30, 67 (1988).
(50) J. C. Suita, private communication (1995).

(51) Yu. A. Batusov et al., Sov. J. Nucl. Phys. 23, 621 (1976).

(52) P. David et al., Z. Phys. A 328, 37 (1987).

(53)D. M. G. Dautet, Ph. D. thesis, Simon Fraser University, Canada (1980).

(54) B. Budick et al., Phys. Rev. Lett. 24, 604 (1970).

(55) V. E. Viola, Nucl. Data Section A1 (No. 5), 391(1966).

(56) P. E. Haustein and T. J. Ruth, Phys. Rev. C 18, 2241 (1978).

(57) V. M. Abazov et al., Nucl. Phys. A 274, 463 (1976).

(58) J. P. Bocquet et al., Z. Phys. A 342, 183 (1992).

(59) J. A. Lopez and J. Randrup, Nucl. Phys. A 491, 477 (1989).

(60) A. S. Iljinov et al., Phys. Rev. C 39, 1420(1989).

(61) I. V. Ryzhov et al., Nucl. Instrum. Methods Phys. Res. A 562, 439 (2006).

(62) C. Cetina et al., Phys. Rev. C 65, 044622 (2002).

(63) J. Cugnon and J. Vandermeulen, Nucl. Phys. A 445, 717 (1985).

(64) A. Fluckiger et al., SIN Jahrsbericht 1975, unpublished; data shown in E. Gadioli et al., Z. Phys. A 288, 39 (1978).

(65) E. Gadioli, E. Gadioli-Erba, and A. Moroni, 1978, Z. Phys. A 288, 39 (1978).

(66)L. Husain and S. Katkoff, Phys. Rev. C 4, 263(1971).

(67) V. Lucherini et al., Phys. Rev. C 39, 911 (1989).

(68) D. I. Ivanov et al., Z. Phys. A 352, 191(1995).

(69) S. Schmid et al., Z. Phys. A 359, 27 (1997).

(70) J. R. Nix and E. Sassi, Nucl. Phys. 81, 61 (1966).

(71) A. S. Iljinov, V. I. Nazaruk, and S. E. Chigrinov, Nucl. Phys. A 268, 513 (1976).

(72) D. A. De Lima, J. B. Martins, and O. A. P. Tavares, Nuovo Cimento 103, A 701 (1990).

(73) H. A. Khan and N. A. Khan, Phys. Rev. C 29, 2199 (1984).

(74) L. G. Moretto et al., Phys. Rev. C 44, 2644 (1991).

(75)L. G. Moretto, D. N. Dels, and G. J. Wozniak, Phys. Rev. Lett. 71, 3935 (1993).

(76) N. C. Mukhopadhyay, J. Hadermann, and K. Junker, Nucl. Phys. A 318, 448 (1979).

(77) M. Blann and J. Bisplinghoff, Lawrence Livermore National Laboratory Report UCID-19614 (1982).

(78)Z. Fraenkel, E. Piasetzky, and G. Kalbermann, Phys. Rev. C 26, 1618 (1982).

(79) A. V. Prokofiev, S. G. Mashnik, and A. J. Sierk, Nucl. Science Engin. 131, 78 (1999).

(80)Z. Yasin et al., to be published.

(81) S. G. Mashnik, private communication.

(82) J. P. Bondorf et al., Nucl. Phys. A 443, 321; A 444, 460 (1985).

(83) R. Donangelo and H. Schechter, private communication (1990). 